\title{
GENERALIZED CONFORMAL DENSITIES FOR HIGHER PRODUCTS OF RANK ONE HADAMARD SPACES
}

\author{
GaBRIELE LinK
}

April 24, 2022

\begin{abstract}
Let $X$ be a product of locally compact rank one Hadamard spaces and $\Gamma$ a discrete group of isometries which contains two elements projecting to a pair of independent rank one isometries in each factor. In Lin13 we gave a precise description of the structure of the geometric limit set $L_{\Gamma}$ of $\Gamma$; our aim in this paper is to describe this set from a measure theoretical point of view, using as a basic tool the properties of the exponent of growth of $\Gamma$ established in the aforementioned article. We first show that the conformal density obtained from the classical Patterson-Sullivan construction is supported in a unique $\Gamma$-invariant subset of the geometric limit set; generalizing this classical construction we then obtain measures supported in each $\Gamma$-invariant subset of the regular limit set and investigate their properties.

We remark that apart from Kac-Moody groups over finite fields acting on the Davis complex of their associated twin building, the probably most interesting examples to which our results apply are isometry groups of reducible CAT(0)-cube complexes without Euclidean factors.
\end{abstract}

\section{Introduction}

Let $(X, d)$ be a product of $r$ locally compact Hadamard spaces $\left(X_{i}, d_{i}\right)$ endowed with the $\ell^{2}$-metric, which makes $X$ itself a locally compact Hadamard space, i.e. a locally compact complete simply connected metric spaces of non-positive Alexandrov curvature. It is well-known that every locally compact Hadamard space can be compactified by adding its geometric boundary $\partial X$ endowed with the cone topology (see Bal95, Chapter II]). If $X$ is a product space, then the regular geometric boundary $\partial X^{\text {reg }}$ of $X$ - which consists of the set of equivalence classes of geodesic rays which do not project to a point in one of the factors - is a dense open subset of $\partial X$ homeomorphic to the Cartesian product of the geometric boundaries $\partial X_{i}$ of the factors $X_{i}$ (which we call the Furstenberg boundary $\partial^{F} X$ of $X$ ) times a factor $E^{+}=\left\{\theta \in \mathbb{R}_{>0}^{r}:\|\theta\|=1\right\}$; the projection to the last factor will be called the slope of a point in $\partial X^{r e g}$. Every point $\tilde{\eta}$ in the singular geometric boundary $\partial X^{\text {sing }}=\partial X \backslash \partial X^{\text {reg }}$ similarly has a well-defined slope $\theta=\left(\theta_{1}, \theta_{2}, \ldots, \theta_{r}\right) \in E:=\left\{\theta \in \mathbb{R}_{\geq 0}^{r}:\|\theta\|=1\right\}$ such that $\theta_{i}=0$ for at least 
one $i \in\{1,2, \ldots, r\}$; notice that in this case the projection of $\tilde{\eta}$ to $\partial X_{i}$ is not welldefined and $\tilde{\eta}$ is already completely determined by its slope and its projections $\eta_{i}$ to the geometric boundaries $\partial X_{i}$ of the factors $X_{i}$ for which $\theta_{i}>0$.

For a group $\Gamma \subset \operatorname{Is}\left(X_{1}\right) \times \operatorname{Is}\left(X_{2}\right) \times \cdots \times \operatorname{Is}\left(X_{r}\right)$ acting properly discontinuously by isometries on $X$ the limit set is defined by $L_{\Gamma}:=\overline{\Gamma \cdot x} \cap \partial X$, where $x \in X$ is arbitrary. In order to relate the critical exponent of a Fuchsian group to the Hausdorff dimension of its limit set, S. J. Patterson ( [Pat76]) and D. Sullivan ( [Sul79]) developed a theory of conformal densities. It turned out that for higher rank symmetric spaces and Euclidean buildings these densities in general detect only a small part of the geometric limit set (see [Alb99]). In order to measure the limit set in each invariant subset of the limit set, a class of generalized conformal densities were independently introduced in Qui02 and Lin04. One of the main goals in this paper is to adapt this construction to discrete groups $\Gamma \subset \operatorname{Is}\left(X_{1}\right) \times \operatorname{Is}\left(X_{2}\right) \times \cdots \times \operatorname{Is}\left(X_{r}\right)$ which contain a pair of isometries projecting to independent rank one elements in each factor. Related questions were considered by M. Burger ( [Bur93]) for graphs of convex cocompact groups in a product of rank one symmetric spaces, and by F. Dal'bo and I. Kim ( [DK08]) for discrete isometry groups of a product of two Hadamard manifolds of pinched negative curvature.

One important class of examples satisfying our conditions are Kac-Moody groups $\Gamma$ over a finite field which act by isometries on a product $X=X_{1} \times X_{2}$, the CAT(0)realization of the associated twin building $\mathcal{B}_{+} \times \mathcal{B}_{-}$. Indeed, there exists an element $h=\left(h_{1}, h_{2}\right)$ projecting to a rank one element in each factor by Remark 5.4 and the proof of Corollary 1.3 in [CF10]. Moreover, the action of the Weyl group produces many axial isometries $g=\left(g_{1}, g_{2}\right)$ with $g_{i}$ rank one and independent from $h_{i}$ for $i=1,2$. Notice that if the order of the ground field is sufficiently large, then $\Gamma \subset \operatorname{Is}\left(X_{1}\right) \times \operatorname{Is}\left(X_{2}\right)$ is an irreducible lattice (see e.g. [Rém99] and [CR09]).

Moreover, according to the Rank Rigidity Theorem ( [CS11, Theorem A]) every finite-dimensional CAT(0)-cube complex $X$ admitting a group $\Gamma$ of automorphisms without fixed point in the geometric compactification of $X$ and without a rank one isometry possesses a convex $\Gamma$-invariant subcomplex which is a product of two unbounded cube subcomplexes; so one inductively gets a convex $\Gamma$-invariant subcomplex of $X$ which can be decomposed into a finite product of rank one Hadamard spaces. In particular, our results apply to reducible finite-dimensional CAT(0)-cube complexes without Euclidean factor and discrete isometry groups as above.

Apart from these examples possible factors of $X$ include locally compact Hadamard spaces of strictly negative Alexandrov curvature (compare [DK08] in the manifold setting). In this special case every non-elliptic and non-parabolic isometry in one of the factors is already a rank one element. Prominent examples here which are already covered by the above mentioned results of J. F. Quint and the author are Hilbert modular groups acting as irreducible lattices on a product of hyperbolic planes and graphs of convex cocompact groups of rank one symmetric spaces (see also [Bur93]).

A central role throughout the paper is played by the exponent of growth of $\Gamma$ of given slope $\theta=\left(\theta_{1}, \theta_{2}, \ldots, \theta_{r}\right) \in E$ introduced in Section 7 of [Lin13. To recall its definition we fix a point $x=\left(x_{1}, x_{2}, \ldots, x_{r}\right)$ in $X, \varepsilon>0, n \gg 1$ and consider the 
cardinality $N_{\theta}^{\varepsilon}(n)$ of the set

$$
\begin{aligned}
\left\{\gamma=\left(\gamma_{1}, \gamma_{2}, \ldots, \gamma_{r}\right) \in \Gamma: 0<d(x, \gamma x)<n,\right. \\
\left.\left.\qquad\left|\frac{d_{i}\left(x_{i}, \gamma_{i} x_{i}\right)}{d(x, \gamma x)}-\theta_{i}\right|<\varepsilon \text { for all } 1 \leq i \leq r\right\}\right\} .
\end{aligned}
$$

This number counts all orbit points $\gamma x$ of distance less than $n$ to the point $x$ which in addition are "close" to a geodesic ray in the class of a boundary point with slope $\theta$.

Definition 1.1 The exponent of growth of $\Gamma$ of slope $\theta \in E$ is defined by

$$
\delta_{\theta}(\Gamma):=\lim _{\varepsilon \rightarrow 0}\left(\limsup _{n \rightarrow \infty} \frac{\ln N_{\theta}^{\varepsilon}(n)}{n}\right) .
$$

The quantity $\delta_{\theta}(\Gamma)$ can be thought of as a function of $\theta \in E$ which describes the exponential growth rate of orbit points converging to limit points of slope $\theta$. It is an invariant of $\Gamma$ which carries more information than the critical exponent $\delta(\Gamma)$; from Theorem 7.6 in [Lin13] (compare also [Lin10, Theorem 7.4] in the case of only two factors) it follows that there exists a unique slope $\theta^{*} \in E$ such that the exponent of growth of $\Gamma$ is maximal for this slope and equal to the critical exponent $\delta(\Gamma)$.

Our first result concerns the measures on the geometric boundary obtained by the classical Patterson-Sullivan construction. Analogous to the case of symmetric spaces or Euclidean buildings of higher rank we have the following result:

Theorem A The Patterson-Sullivan construction produces a conformal density with support in a single $\Gamma$-invariant subset of the geometric limit set. Every point in its support has slope $\theta^{*}$ as above.

Thus in order to measure the remaining $\Gamma$-invariant subsets of the limit set, we need a more sophisticated construction. Inspired by the paper [Bur93] of M. Burger we will consider densities with more degrees of freedom than the classical conformal density.

Before we can state the remaining results we need more definitions. We fix a base point $o=\left(o_{1}, o_{2}, \ldots, o_{r}\right) \in X$. For $\theta \in E$ we denote $\partial X_{\theta}$ the set of points in the geometric boundary of slope $\theta$ and $I^{+}(\theta)=\left\{i \in\{1,2, \ldots, r\}: \theta_{i}>0\right\}$. Then according to the remark at the end of the first paragraph the strata $\partial X_{\theta}$ is homeomorphic to the Cartesian product of the geometric boundaries $\partial X_{i}$ with $i \in I^{+}(\theta)$; for $\theta \in E^{+}$ this is obviously the whole Furstenberg boundary $\partial^{F} X=\partial X_{1} \times \partial X_{2} \times \cdots \times \partial X_{r}$. For a point $\tilde{\eta} \in \partial X_{\theta}$ and $i \in I^{+}(\theta)$ we will denote $\eta_{i} \in \partial X_{i}$ the projection to the factor $\partial X_{i}$. Moreover, if $i \in\{1,2, \ldots, r\}$ and $\eta_{i} \in \partial X_{i}$ we let $\beta_{\eta_{i}}\left(\cdot, o_{i}\right)$ denote the Busemann function centered at $\eta_{i}$ based at $o_{i}$.

Definition 1.2 Let $\mathcal{M}^{+}(\partial X)$ denote the cone of positive finite Borel measures on $\partial X, \theta=\left(\theta_{1}, \theta_{2}, \ldots, \theta_{r}\right) \in E$ and $b=\left(b_{1}, b_{2}, \ldots, b_{r}\right) \in \mathbb{R}^{r}$ such that $b_{i}=0$ for all $i \in\{1,2, \ldots, r\} \backslash I^{+}(\theta)$. A $\Gamma$-invariant $(b, \theta)$-density is a map

$$
\begin{array}{ccc}
\mu: X & \rightarrow & \mathcal{M}^{+}(\partial X) \\
x & \mapsto & \mu_{x}
\end{array}
$$

such that for any $x=\left(x_{1}, x_{2}, \ldots, x_{r}\right) \in X$ the following three properties hold: 
(i) $\emptyset \neq \operatorname{supp}\left(\mu_{x}\right) \subset L_{\Gamma} \cap \partial X_{\theta}$,

(ii) $\forall \gamma \in \Gamma \quad \gamma_{*} \mu_{x}=\mu_{\gamma x}{ }^{1}$,

(iii) $\forall \tilde{\eta} \in \operatorname{supp}\left(\mu_{o}\right)$

$$
\frac{d \mu_{x}}{d \mu_{o}}(\tilde{\eta})=\mathrm{e}^{b_{1} \beta_{\eta_{1}}\left(o_{1}, x_{1}\right)+b_{2} \beta_{\eta_{2}}\left(o_{2}, x_{2}\right)+\cdots+b_{r} \beta_{\eta_{r}}\left(o_{r}, x_{r}\right)} .
$$

Notice that if $\theta_{i}=0$ for some $i \in\{1,2, \ldots, r\}$, then for $\tilde{\eta} \in \partial X_{\theta}$ the projection $\eta_{i} \in \partial X_{i}$ is not defined; however, the condition $b_{i}=0$ ensures that the exponent in (iii) is well-defined. Moreover, the conformal density from Theorem A is a special case of such a density with support in $\partial X_{\theta^{*}}$ and parameters $b_{i}=\delta(\Gamma) \cdot \theta_{i}^{*}, i \in\{1,2, \ldots, r\}$.

We next give a criterion for the existence of a $(b, \theta)$-density.

Theorem $\mathbf{B}$ If $\theta \in E^{+}$is such that $\delta_{\theta}(\Gamma)>0$, then there exists a $(b, \theta)$-density for some parameters $b=\left(b_{1}, b_{2}, \ldots, b_{r}\right) \in \mathbb{R}^{r}$.

Notice that according to Theorem 7.9 of [Lin13] we have $\delta_{\theta}(\Gamma)>0$ for $\theta$ in the relative interior of the intersection of the limit cone $\ell_{\Gamma}$ with the vector subspace of $\mathbb{R}^{r}$ it spans. In Section [ 6 we will give an explicit construction of the $(b, \theta)$-density from Theorem B above.

The following results about $(b, \theta)$-densities in particular apply to any conformal density supported in a single $\Gamma$-invariant subset of the geometric limit set, not only the one obtained by the classical Patterson-Sullivan construction. Our main tool is a so-called shadow lemma for $(b, \theta)$-densities, which is a generalization of the well-known shadow lemma for conformal densities. It first gives a condition for the parameters of a $(b, \theta)$-density in terms of the exponent of growth.

Theorem C If a $\Gamma$-invariant $(b, \theta)$-density exists for some $\theta=\left(\theta_{1}, \theta_{2}, \ldots, \theta_{r}\right) \in E^{+}$, then

$$
\delta_{\theta}(\Gamma) \leq \sum_{i=1}^{r} b_{i} \cdot \theta_{i} .
$$

The following subsets of the geometric limit set will play an important role in the sequel.

Definition 1.3 A point $\tilde{\xi} \in \partial X_{\theta}$ is called a radial limit point of $\Gamma$ if there exists a sequence $\left(\gamma_{n}\right)=\left(\left(\gamma_{n, 1}, \gamma_{n, 2}, \ldots, \gamma_{n, r}\right)\right) \subset \Gamma$ such that $\gamma_{n}$ o converges to $\tilde{\xi}$ and such that for all $i \in I^{+}(\theta)$ the sequence $\gamma_{n, i} O_{i} \subset X_{i}$ stays at bounded distance of one (and hence any) geodesic ray in the class of $\xi_{i} \subset \partial X_{i}$.

We will denote the set of all radial limit points of $\Gamma$ by $L_{\Gamma}^{r a d}$.

Notice that in general a radial limit point $\tilde{\xi} \in \partial X$ is not approached by a sequence $\gamma_{n} O \subset X$ staying at bounded distance of a geodesic ray in the class of $\tilde{\xi}$.

Our next statement shows that for certain $(b, \theta)$-densities the corresponding exponent of growth $\delta_{\theta}(\Gamma)$ is completely determined by the parameters $\theta \in E^{+}$and $b=\left(b_{1}, b_{2}, \ldots, b_{r}\right) \in \mathbb{R}^{r}:$

\footnotetext{
${ }^{1}$ Here $\gamma_{*} \mu_{x}$ denotes the measure defined by $\gamma_{*} \mu_{x}(E)=\mu_{x}\left(\gamma^{-1} E\right)$ for any Borel set $E \subset \partial X$
} 
Theorem $\mathbf{D}$ If $\theta=\left(\theta_{1}, \theta_{2}, \ldots, \theta_{r}\right) \in E^{+}$, and $\mu$ is a $\Gamma$-invariant $(b, \theta)$-density which gives positive measure to the radial limit set, then

$$
\delta_{\theta}(\Gamma)=\sum_{i=1}^{r} b_{i} \cdot \theta_{i} .
$$

The following theorem gives a restriction for the atomic part of our measures.

Theorem $\mathbf{E}$ If $\theta \in E^{+}$such that $\delta_{\theta}(\Gamma)>0$, and $\mu$ is a $\Gamma$-invariant $(b, \theta)$-density, then a radial limit point is not a point mass for $\mu$.

The paper is organized as follows: In Section 2 we recall basic facts about Hadamard spaces and rank one isometries. Section 3 deals with the product case and provides some tools for the proof of the so-called shadow lemma in Section 7. In Section 4 we introduce and study the properties of the exponent of growth. Section 5 recalls the classical Patterson-Sullivan construction in our setting. The main new result here is Theorem A. In Section [6] we introduce a generalized Poincaré series that allows to construct $(b, \theta)$-densities, and therefore proves Theorem B. Using the shadow lemma, in Section 7 we deduce properties of $(b, \theta)$-densities and prove Theorems C, D and E.

Acknowledgements: The first draft of this paper was initiated during the author's stay at IHES in Bures-sur-Yvette. She warmly thanks the institute for its hospitality and the inspiring atmosphere.

\section{Preliminaries}

The purpose of this section is to introduce terminology and notation and to summarize basic results about Hadamard spaces and rank one isometries. The main references here are [BH99] and Bal95] (see also [BB95], and [BGS85, Bal82] in the case of Hadamard manifolds).

Let $(X, d)$ be a metric space. A geodesic path joining $x \in X$ to $y \in X$ is a map $\sigma$ from a closed interval $[0, l] \subset \mathbb{R}$ to $X$ such that $\sigma(0)=x, \sigma(l)=y$ and $d\left(\sigma(t), \sigma\left(t^{\prime}\right)\right)=\left|t-t^{\prime}\right|$ for all $t, t^{\prime} \in[0, l]$. We will denote such a geodesic path $\sigma_{x, y} . X$ is called geodesic if any two points in $X$ can be connected by a geodesic path, if this path is unique we say that $X$ is uniquely geodesic. In this text $X$ will be a Hadamard space, i.e. a complete geodesic metric space in which all triangles satisfy the CAT(0)inequality. This implies in particular that $X$ is simply connected and uniquely geodesic. A geodesic or geodesic line in $X$ is a map $\sigma: \mathbb{R} \rightarrow X$ such that $d\left(\sigma(t), \sigma\left(t^{\prime}\right)\right)=\left|t-t^{\prime}\right|$ for all $t, t^{\prime} \in \mathbb{R}$, a geodesic ray is a map $\sigma:[0, \infty) \rightarrow X$ such that $d\left(\sigma(t), \sigma\left(t^{\prime}\right)\right)=\left|t-t^{\prime}\right|$ for all $t, t^{\prime} \in[0, \infty)$. Notice that in the non-Riemannian setting completeness of $X$ does not imply geodesically completeness, i.e. not every geodesic path or ray can be extended to a geodesic.

From here on we will assume that $X$ is a locally compact Hadamard space. The geometric boundary $\partial X$ of $X$ is the set of equivalence classes of asymptotic geodesic rays endowed with the cone topology (see e.g. [Bal95, chapter II]). The action of the 
isometry group $\operatorname{Is}(X)$ on $X$ naturally extends to an action by homeomorphisms on the geometric boundary. Moreover, since $X$ is locally compact, this boundary $\partial X$ is compact and the space $X$ is a dense and open subset of the compact space $\bar{X}:=X \cup \partial X$. For $x \in X$ and $\xi \in \partial X$ arbitrary there exists a geodesic ray emanating from $x$ which belongs to the class of $\xi$. We will denote such a ray $\sigma_{x, \xi}$.

We say that two points $\xi, \eta \in \partial X$ can be joined by a geodesic if there exists a geodesic $\sigma: \mathbb{R} \rightarrow X$ such that $\sigma(-\infty)=\xi$ and $\sigma(\infty)=\eta$. It is well-known that if $X$ is $\operatorname{CAT}(-1)$, i.e. of negative Alexandrov curvature bounded above by -1 , then every pair of distinct points in the geometric boundary can be joined by a geodesic. This is not true in the general CAT(0)-case.

Let $x, y \in X, \xi \in \partial X$ and $\sigma$ a geodesic ray in the class of $\xi$. We set

$$
\beta_{\xi}(x, y):=\lim _{s \rightarrow \infty}(d(x, \sigma(s))-d(y, \sigma(s))) .
$$

This number is independent of the chosen ray $\sigma$, and the function

$$
\begin{aligned}
\beta_{\xi}(\cdot, y): \quad X & \rightarrow \mathbb{R} \\
x & \mapsto \beta_{\xi}(x, y)
\end{aligned}
$$

is called the Busemann function centered at $\xi$ based at $y$ (see also [Bal95, chapter II). From the definition one immediately gets the following properties of the Busemann function:

$$
\begin{array}{rlrl}
\left|\beta_{\xi}(x, y)\right| & \leq d(x, y) & \\
\beta_{\xi}(x, y) & =-\beta_{\xi}(y, x) & \text { (anti-symmetry) } \\
\beta_{\xi}(x, z) & =\beta_{\xi}(x, y)+\beta_{\xi}(y, z) & & \text { (cocycle identity) } \\
\beta_{\xi}(x, y) & =\beta_{g \cdot \xi}(g \cdot x, g \cdot y) & & (\mathrm{Is}(X) \text {-invariance) }
\end{array}
$$

for all $x, y, z \in X, \xi \in \partial X$ and $g \in \operatorname{Is}(X)$. Moreover, $\beta_{\xi}(x, y)=d(x, y)$ if and only if $y$ is a point on the geodesic ray $\sigma_{x, \xi}$, and we have the following easy

Lemma 2.1 Let $c>0, x, z \in X$ and $\xi \in \partial X$ such that $d\left(z, \sigma_{x, \xi}\right)<c$. Then

$$
0 \leq d(x, z)-\beta_{\xi}(x, z)<2 c .
$$

Proof. The first inequality follows from $\left|\beta_{\xi}(x, y)\right| \leq d(x, y)$. For the second one let $y \in X$ be a point on the geodesic ray $\sigma_{x, \xi}$ such that $d(z, y)<c$. Then for all $s>d(x, y)$ we have by the triangle inequality

$$
\begin{aligned}
d\left(x, \sigma_{x, \xi}(s)\right)-d\left(z, \sigma_{x, \xi}(s)\right) & \geq d\left(x, \sigma_{x, \xi}(s)\right)-d(z, y)-d\left(y, \sigma_{x, \xi}(s)\right) \\
& =d(x, y)-d(z, y)>d(x, y)-c
\end{aligned}
$$

hence $\quad d(x, z)-\beta_{\xi}(x, z) \leq d(x, y)+c-d(x, y)+c=2 c$.

A geodesic $\sigma: \mathbb{R} \rightarrow X$ is said to bound a flat half-plane if there exists a closed convex subset $\iota([0, \infty) \times \mathbb{R})$ in $X$ isometric to $[0, \infty) \times \mathbb{R}$ such that $\sigma(t)=\iota(0, t)$ for all 
$t \in \mathbb{R}$. Similarly, a geodesic $\sigma: \mathbb{R} \rightarrow X$ bounds a flat strip of width $c>0$ if there exists a closed convex subset $\iota([0, c] \times \mathbb{R})$ in $X$ isometric to $[0, c] \times \mathbb{R}$ such that $\sigma(t)=\iota(0, t)$ for all $t \in \mathbb{R}$. We call a geodesic $\sigma: \mathbb{R} \rightarrow X$ a rank one geodesic if $\sigma$ does not bound a flat half-plane.

The following important lemma states that even though we cannot join any two distinct points in the geometric boundary of $X$, given a rank one geodesic we can at least join points in a neighborhood of its extremities. More precisely, we have the following well-known

Lemma 2.2 ( Bal95], Lemma III.3.1) Let $\sigma: \mathbb{R} \rightarrow X$ be a rank one geodesic. Then there exist $c>0$ and neighborhoods $U^{-}, U^{+}$of $\sigma(-\infty), \sigma(\infty)$ in $\bar{X}$ such that for any $\xi \in U^{-}$and $\eta \in U^{+}$there exists a rank one geodesic joining $\xi$ and $\eta$. For any such geodesic $\sigma^{\prime}$ we have $d\left(\sigma^{\prime}, \sigma(0)\right) \leq c$.

The following kind of isometries will play a central role in the sequel.

Definition 2.3 An isometry $h$ of $X$ is called axial, if there exists a constant $l=l(h)>0$ and a geodesic $\sigma$ such that $h(\sigma(t))=\sigma(t+l)$ for all $t \in \mathbb{R}$. We call $l(h)$ the translation length of $h$, and $\sigma$ an axis of $h$. The boundary point $h^{+}:=\sigma(\infty)$ is called the attractive fixed point, and $h^{-}:=\sigma(-\infty)$ the repulsive fixed point of $h$. We further set $A x(h):=\{x \in X: d(x, h x)=l(h)\}$.

We remark that $\operatorname{Ax}(h)$ consists of the union of parallel geodesics translated by $h$, and $\overline{\operatorname{Ax}(h)} \cap \partial X$ is exactly the set of fixed points of $h$.

DEFINITION 2.4 An axial isometry is called rank one if it possesses a rank one axis. Two rank one isometries are called independent, if their fixed point sets are disjoint.

Notice that if $h$ is rank one, then $h^{+}$and $h^{-}$are the only fixed points of $h$. Let us recall the north-south dynamics of rank one isometries.

Lemma 2.5 ([Bal95], Lemma III.3.3) Let $h$ be a rank one isometry. Then

(a) any $\xi \in \partial X \backslash\left\{h^{+}\right\}$can be joined to $h^{+}$by a geodesic, and every geodesic joining $\xi$ to $h^{+}$is rank one,

(b) given neighborhoods $U^{-}$of $h^{-}$and $U^{+}$of $h^{+}$in $\bar{X}$ there exists $N_{0} \in \mathbb{N}$ such that $h^{-n}\left(\bar{X} \backslash U^{+}\right) \subset U^{-}$and $h^{n}\left(\bar{X} \backslash U^{-}\right) \subset U^{+}$for all $n \geq N_{0}$.

If $\Gamma$ is a group acting by isometries on a locally compact Hadamard space $X$ we define its geometric limit set by $L_{\Gamma}:=\overline{\Gamma \cdot x} \cap \partial X$, where $x \in X$ is arbitrary.

\section{Products of Hadamard spaces}

Now consider $r$ locally compact Hadamard spaces $\left(X_{1}, d_{1}\right),\left(X_{2}, d_{2}\right), \ldots,\left(X_{r}, d_{r}\right)$ and their Cartesian product $X=X_{1} \times X_{2} \times \cdots \times X_{r}$ endowed with the distance $d=\sqrt{d_{1}^{2}+d_{2}^{2}+\cdots+d_{r}^{2}}$. Notice that $(X, d)$ is again a locally compact Hadamard space. 
We denote $\mathbb{R}_{\geq 0}^{r}:=\left\{\left(t_{1}, t_{2}, \ldots, t_{r}\right) \in \mathbb{R}^{r}: t_{i} \geq 0\right.$ for all $\left.i \in\{1,2, \ldots, r\}\right\}$ and $\mathbb{R}_{>0}^{r}:=\left\{\left(t_{1}, t_{2}, \ldots, t_{r}\right) \in \mathbb{R}^{r}: t_{i}>0\right.$ for all $\left.i \in\{1,2, \ldots, r\}\right\}$. To any pair of points $x=\left(x_{1}, x_{2}, \ldots, x_{r}\right), z=\left(z_{1}, z_{2}, \ldots, z_{r}\right) \in X$ we associate the vector

$$
H(x, z):=\left(\begin{array}{c}
d_{1}\left(x_{1}, z_{1}\right) \\
d_{2}\left(x_{2}, z_{2}\right) \\
\vdots \\
d_{r}\left(x_{r}, z_{r}\right)
\end{array}\right) \in \mathbb{R}_{\geq 0}^{r}
$$

which we call the distance vector of the pair $(x, z)$. Notice that if $\|\cdot\|$ denotes the Euclidean norm in $\mathbb{R}^{r}$, we clearly have $\|H(x, z)\|=d(x, z)$. For $z \neq x$ we further define the direction of $z$ with respect to $x$ by the unit vector

$$
\widehat{H}(x, z):=\frac{H(x, z)}{d(x, z)} \in \mathbb{R}_{\geq 0}^{r} .
$$

The following lemma is immediate and states that distance vectors and directions are invariant by $\operatorname{Is}\left(X_{1}\right) \times \operatorname{Is}\left(X_{2}\right) \times \cdots \times \operatorname{Is}\left(X_{r}\right)$.

Lemma 3.1 If $g=\left(g_{1}, g_{2}, \ldots, g_{r}\right) \in I s\left(X_{1}\right) \times I s\left(X_{2}\right) \times \cdots \times I s\left(X_{r}\right), x=\left(x_{1}, x_{2}, \ldots, x_{r}\right)$, $z=\left(z_{1}, z_{2}, \ldots, z_{r}\right) \in X$, then

$$
H(g x, g z)=H(x, z) \quad \text { and } \quad \widehat{H}(g x, g z)=\widehat{H}(x, z) .
$$

Denote $p_{i}: X \rightarrow X_{i}, i \in\{1,2, \ldots, r\}$, the natural projections. Every geodesic path $\sigma:[0, l] \rightarrow X$ can be written as a product $\sigma(t)=\left(\sigma_{1}\left(t \cdot \theta_{1}\right), \sigma_{2}\left(t \cdot \theta_{2}\right), \ldots, \sigma_{r}\left(t \cdot \theta_{r}\right)\right)$, where $\sigma_{i}$ are geodesic paths in $X_{i}, i=1,2, \ldots r$, and the $\theta_{i} \geq 0$ satisfy

$$
\sum_{i=1}^{r} \theta_{i}^{2}=1 .
$$

The unit vector

$$
\operatorname{sl}(\sigma):=\left(\begin{array}{c}
\theta_{1} \\
\theta_{2} \\
\vdots \\
\theta_{r}
\end{array}\right) \in E:=\left\{\theta \in \mathbb{R}_{\geq 0}^{r}:\|\theta\|=1\right\}
$$

equals the direction of the points $\sigma(t), t \in(0, l]$, with respect to $\sigma(0)$ and is called the slope of $\sigma$. We say that a geodesic path $\sigma$ is regular if its slope does not possess a coordinate zero, i.e. if

$$
\operatorname{sl}(\sigma) \in E^{+}:=\left\{\theta \in \mathbb{R}_{>0}^{r}:\|\theta\|=1\right\}
$$

otherwise $\sigma$ is said to be singular. In other words, $\sigma$ is regular if none of the projections $p_{i}(\sigma([0, l])), i \in\{1,2, \ldots, r\}$, is a point. 
If $x \in X$ and $\sigma:[0, \infty) \rightarrow X$ is an arbitrary geodesic ray, then by elementary geometric estimates one has the relation

$$
\operatorname{sl}(\sigma)=\lim _{t \rightarrow \infty} \widehat{H}(x, \sigma(t))=\lim _{t \rightarrow \infty} \frac{H(x, \sigma(t))}{d(x, \sigma(t))}
$$

between the slope of $\sigma$ and the directions of $\sigma(t), t>0$, with respect to $x$. Similarly, one can easily show that any two geodesic rays representing the same (possibly singular) point in the geometric boundary necessarily have the same slope. So we may define the slope $\operatorname{sl}(\tilde{\xi})$ of a point $\tilde{\xi} \in \partial X$ as the slope of an arbitrary geodesic ray representing $\tilde{\xi}$. The regular geometric boundary $\partial X^{r e g}$ and the singular geometric boundary $\partial X^{\text {sing }}$ of $X$ are then naturally defined by

$$
\partial X^{r e g}:=\left\{\tilde{\xi} \in \partial X: \operatorname{sl}(\tilde{\xi}) \in E^{+}\right\}, \quad \partial X^{\text {sing }}:=\partial X \backslash \partial X^{\text {sing }}
$$

the singular boundary $\partial X^{\text {sing }}$ consists of equivalence classes of geodesic rays in $X$ which project to a point in at least one of the factors $X_{i}$. More precisely, given $\theta=\left(\theta_{1}, \theta_{2}, \ldots, \theta_{r}\right) \in E$, we can define the subset

$$
\partial X_{\theta}:=\{\tilde{\xi} \in \partial X: \operatorname{sl}(\tilde{\xi})=\theta\}
$$

of the geometric boundary; we further denote $I^{+}(\theta):=\left\{i \in\{1,2, \ldots, r\}: \theta_{i}>0\right\}$. It is easy to see that two geodesic rays $\sigma, \sigma^{\prime}$ in $X$ represent the same point in $\partial X_{\theta}$ if and only if $\sigma_{i}(\infty)=\sigma_{i}^{\prime}(\infty)$ for all $i \in I^{+}(\theta)$. Hence $\partial X_{\theta}$ is homeomorphic to the Cartesian product of the geometric boundaries $\partial X_{i}$ with $i \in I^{+}(\theta)$.

We further remark that a sequence $\left(y_{n}\right)=\left(\left(y_{n, 1}, y_{n, 2}, \ldots, y_{n, r}\right)\right) \subset X$ converges to a point $\tilde{\eta} \in \partial X_{\theta}$ if and only if $y_{n, i} \rightarrow \eta_{i}$ for all $i \in I^{+}(\theta)$ and $\widehat{H}\left(x, y_{n}\right) \rightarrow \theta$ as $n \rightarrow \infty$ for some (and hence any) fixed $x \in X$.

For higher rank symmetric spaces and Bruhat-Tits buildings there is a well-known notion of Furstenberg boundary which - for a product of rank one spaces - coincides with the product of the geometric boundaries. In our setting we choose to call the product $\partial X_{1} \times \partial X_{2} \times \cdots \times \partial X_{r}$ endowed with the product topology the Furstenberg boundary $\partial^{F} X$ of $X$. Since $\partial X^{r e g}$ is homeomorphic to $\partial^{F} X \times E^{+}$we have a natural projection

$$
\begin{aligned}
& \pi^{F}: \quad \partial X^{r e g} \quad \rightarrow \quad \partial^{F} X \\
& \left(\xi_{1}, \xi_{2}, \ldots, \xi_{r}, \theta\right) \mapsto\left(\xi_{1}, \xi_{2}, \ldots, \xi_{r}\right)
\end{aligned}
$$

and a natural action of the group $\operatorname{Is}\left(X_{1}\right) \times \operatorname{Is}\left(X_{2}\right) \times \cdots \times \operatorname{Is}\left(X_{r}\right)$ by homeomorphisms on the Furstenberg boundary of $X$.

We say that two points $\xi=\left(\xi_{1}, \xi_{2}, \ldots, \xi_{r}\right), \eta=\left(\eta_{1}, \eta_{2}, \ldots, \eta_{r}\right) \in \partial^{F} X$ are opposite if $\xi_{i}$ and $\eta_{i}$ can be joined by a geodesic in $X_{i}$ for all $i \in\{1,2, \ldots, r\}$.

For $x=\left(x_{1}, x_{2}, \ldots, x_{r}\right)$ and $z=\left(z_{1}, z_{2}, \ldots, z_{r}\right) \in X$ such that $z_{i} \neq x_{i}$ for all $i \in\{1,2, \ldots, r\}$, the set

$$
\begin{aligned}
& \mathcal{C}_{x, z}:=\left\{\left(\sigma_{x_{1}, z_{1}}\left(t_{1}\right), \sigma_{x_{2}, z_{2}}\left(t_{2}\right), \ldots,\right.\right.\left.\sigma_{x_{r}, z_{r}}\left(t_{r}\right)\right) \in X: \\
&\left.0 \leq t_{i} \leq d_{i}\left(x_{i}, z_{i}\right) \quad \text { for } \quad i \in\{1,2, \ldots, r\}\right\}
\end{aligned}
$$


is called the Weyl chamber from $x$ to $z$. Notice that if $z_{i}=x_{i}$ for some $i \in\{1,2, \ldots, r\}$, then $\sigma_{x_{i}, z_{i}}$ is not defined, so the assignment in (5) is not well-defined. In this case we set $I(x, z):=\left\{i \in\{1,2, \ldots, r\}: x_{i}=z_{i}\right\}$ and define $\mathcal{C}_{x, z}$

$$
\begin{aligned}
\mathcal{C}_{x, z}:=\left\{\left(y_{1}, y_{2}, \ldots, y_{r}\right) \in X:\right. & y_{i} \in X_{i} \quad \text { arbitrary if } i \in I(x, z), \\
y_{i}=\sigma_{x_{i}, z_{i}}\left(t_{i}\right) & \text { with } \left.\quad 0 \leq t_{i} \leq d_{i}\left(x_{i}, z_{i}\right) \quad \text { if } i \in\{1,2, \ldots, r\} \backslash I(x, z)\right\} .
\end{aligned}
$$

We remark that in the degenerated case $z=x$ our definition gives $\mathcal{C}_{x, x}=\{x\}$.

Similarly, for $x=\left(x_{1}, x_{2}, \ldots, x_{r}\right) \in X, \theta \in E$ and $\tilde{\xi} \in \partial X_{\theta}$ we call

$$
\begin{aligned}
\mathcal{C}_{x, \tilde{\xi}}:=\left\{\left(y_{1}, y_{2}, \ldots, y_{r}\right) \in X:\right. & y_{i}=\sigma_{x_{i}, \xi_{i}}\left(t_{i}\right) \text { with } t_{i} \geq 0 \quad \text { if } i \in I^{+}(\theta), \\
y_{i} & \left.\in X_{i} \text { arbitrary if } i \in\{1,2, \ldots, r\} \backslash I^{+}(\theta)\right\}
\end{aligned}
$$

the Weyl chamber with apex $x$ in the class of $\tilde{\xi}$. In this way we have defined $\mathcal{C}_{x, z}$ for any $x \in X$ and $z \in \bar{X}$. Notice that while Weyl chambers in the class of a regular boundary point are homeomorphic to $\mathbb{R}_{\geq 0}^{r}$, a Weyl chamber in the class of a singular boundary point is much bigger.

The Weyl chamber shadow of a set $B \subset X$ viewed from $x=\left(x_{1}, x_{2}, \ldots, x_{r}\right) \in X \backslash B$ is defined by

$$
\operatorname{Sh}(x: B):=\left\{z \in \bar{X}: p_{i}(z) \neq x_{i} \quad \text { for all } i \in\{1,2, \ldots, r\}, \quad \mathcal{C}_{x, z} \cap B \neq \emptyset\right\} .
$$

It consists of the closure in $\bar{X}$ of all Weyl chambers with apex $x$ which intersect $B$ non-trivially. Notice that in view of (6) we have

$$
\operatorname{Sh}(x: B) \cap \partial X_{\theta}=\left\{\tilde{\xi} \in \partial X_{\theta}: \sigma_{x_{i}, \xi_{i}}\left(\mathbb{R}_{\geq 0}\right) \cap p_{i}(B) \neq \emptyset \quad \text { for all } i \in I^{+}(\theta)\right\} .
$$

We next fix a base point $o=\left(o_{1}, o_{2}, \ldots, o_{r}\right) \in X$. For $x \in X$ and $t>0$ we denote by $B_{x}(t)$ the open ball of radius $t$ centered at $x$. If $h \in \operatorname{Is}\left(X_{1}\right) \times \operatorname{Is}\left(X_{2}\right) \times \cdots \times \operatorname{Is}\left(X_{r}\right)$ is such that all projections $h_{i} \in \operatorname{Is}\left(X_{i}\right)$ are axial with translation length $l_{i}(h)>0$, then $h$ is an axial isometry of the product $X=X_{1} \times X_{2} \times \cdots \times X_{r}$ with translation length $l(h)=\sqrt{l_{1}(h)^{2}+l_{2}(h)^{2}+\cdots+l_{r}(h)^{2}}$, and we denote $\widetilde{h^{+}}, \widetilde{h^{-}} \in \partial X$ its attractive and repulsive fixed points. If for $i \in\{1,2, \ldots, r\} \quad h_{i}^{+}, h_{i}^{-} \in \partial X_{i}$ denote the attractive and repulsive fixed points of the projection $h_{i}$, then, since for any point $x \in \operatorname{Ax}(h)$ and all $n \in \mathbb{Z} \backslash\{0\}$

$$
\widehat{H}\left(x, h^{n} x\right)=\left(\frac{l_{1}(h)}{l(h)}, \frac{l_{2}(h)}{l(h)}, \ldots, \frac{l_{r}(h)}{l(h)}\right)=: \widehat{L}(h) \in E^{+}
$$

we get

$$
\widetilde{h^{ \pm}}=\left(h_{1}^{ \pm}, h_{2}^{ \pm}, \ldots, h_{r}^{ \pm}, \widehat{L}(h)\right) \in \partial X^{r e g} .
$$

So for $h^{ \pm}:=\pi^{F}\left(\widetilde{h^{ \pm}}\right)$we have $h^{ \pm}=\left(h_{1}^{ \pm}, h_{2}^{ \pm}, \ldots, h_{r}^{ \pm}\right)$.

The following proposition states that all Weyl chamber shadows of sufficiently large balls are large in the sense that they contain an open set in $\partial X$. This will be crucial in the proof of the shadow lemma. Notice that our idea of proof - which uses Proposition 4.1 of [Lin13] as a key ingredient - also considerably simplifies the proof of the analogous statement for one factor (see [Kni97, Proposition 3.6] and [Lin07, Lemma $3.5])$. 
Proposition 3.2 Assume that $g=\left(g_{1}, g_{2}, \ldots, g_{r}\right)$ and $h=\left(h_{1}, h_{2}, \ldots, h_{r}\right)$ are axial isometries of $I s\left(X_{1}\right) \times I s\left(X_{2}\right) \times \cdots \times I s\left(X_{r}\right)$ such that $g_{i}$ and $h_{i}$ are independent rank one elements in $I s\left(X_{i}\right)$ for all $i \in\{1,2, \ldots, r\}$. Then there exist open neighborhoods $U_{i} \subset \partial X_{i}$ of $h_{i}^{+}, i \in\{1,2, \ldots, r\}$, a finite set $\Lambda$ in the group $\langle g, h\rangle$ generated by $g, h$ and $c_{0}>0$ with the following properties:

If $U:=U_{1} \times U_{2} \times \cdots \times U_{r} \times E^{+} \subset \partial X^{r e g}$ and $t \geq c_{0}$ then for all $y \in X \backslash B_{o}(t)$ there exists $\lambda \in \Lambda$ such that

$$
\lambda U \subset \operatorname{Sh}\left(y: B_{o}(t)\right) .
$$

Moreover, if $\theta \in E$ and $U_{\theta}$ denotes the Cartesian product of the sets $U_{i}$ with $i \in I^{+}(\theta)$, then

$$
\lambda\left(U_{\theta} \times\{\theta\}\right) \subset \operatorname{Sh}\left(y: B_{o}(t)\right) \cap \partial X_{\theta} .
$$

Proof. For $i=1,2, \ldots, r$ and $\eta_{i} \in\left\{g_{i}^{-}, g_{i}^{+}, h_{i}^{-}, h_{i}^{+}\right\}$we let $U_{i}\left(\eta_{i}\right) \subset \bar{X}_{i}$ be an arbitrary sufficiently small neighborhood of $\eta_{i}^{+} \in \partial X_{i}$ with $o_{i} \notin U_{i}\left(\eta_{i}\right)$ such that all $U_{i}\left(\eta_{i}\right)$ are pairwise disjoint in $\bar{X}_{i}$. Upon taking smaller neighborhoods if necessary Lemma 2.2 provides a constant $c>0$ such that for every $i \in\{1,2, \ldots, r\}$ any pair of points in distinct sets $U_{i}\left(\eta_{i}\right)$ can be joined by a rank one geodesic $\sigma_{i} \subset X_{i}$ with $d\left(o_{i}, \sigma_{i}\right) \leq c$. Moreover, according to Lemma 2.5 (b) there exists a constant $N \in \mathbb{N}$ such that for all $i \in\{1,2, \ldots, r\}$

$$
g_{i}^{ \pm N}\left(\bar{X}_{i} \backslash U_{i}\left(g_{i}^{\mp}\right)\right) \subset U_{i}\left(g_{i}^{ \pm}\right), \quad h_{i}^{ \pm N}\left(\bar{X}_{i} \backslash U_{i}\left(h_{i}^{\mp}\right)\right) \subset U_{i}\left(h_{i}^{ \pm}\right) .
$$

We use induction on $r$ to show the existence of a finite set $\Lambda \subset\langle g, h\rangle$ such that for any $y \in X$ one can find $\lambda \in \Lambda$ with

$$
\lambda y \in U_{1}\left(h_{1}^{-}\right) \times U_{2}\left(h_{2}^{-}\right) \times \cdots \times U_{r}\left(h_{r}^{-}\right) .
$$

For $r=1$ we let $y=y_{1} \in \bar{X}_{1}=U_{1}\left(h_{1}^{+}\right) \cup \bar{X}_{1} \backslash U_{1}\left(h_{1}^{+}\right)$be arbitrary. If $y_{1} \in U_{1}\left(h_{1}^{+}\right)$, then from $U_{1}\left(h_{1}^{+}\right) \subset \bar{X}_{1} \backslash U_{1}\left(g_{1}^{+}\right)$and (9) we get $g_{1}^{-N} y_{1} \in U_{1}\left(g_{1}^{-}\right) \subset \bar{X}_{1} \backslash U_{1}\left(h_{1}^{+}\right)$, hence again by (9)

$$
h_{1}^{-N} g_{1}^{-N} y_{1} \in U_{1}\left(h_{1}^{-}\right) .
$$

If $y_{1} \in \bar{X}_{1} \backslash U_{1}\left(h_{1}^{-}\right)$, then (9) directly gives $h_{1}^{-N} y_{1} \in U_{1}\left(h_{1}^{-}\right)$. So for $r=1$ the set $\Lambda_{1}:=\left\{h^{-N} g^{-N}, h^{-N}\right\} \subset\langle g, h\rangle$ is the desired finite set.

Now assume the assertion holds for $r-1$; we claim that it also holds when $r$ factors are involved. By the induction hypothesis there exists a finite set

$$
\Lambda_{r-1} \subset\left\langle\left(g_{1}, g_{2}, \ldots, g_{r-1}\right),\left(h_{1}, h_{2}, \ldots, h_{r-1}\right)\right\rangle<\operatorname{Is}\left(X_{1}\right) \times \operatorname{Is}\left(X_{2}\right) \times \cdots \times \operatorname{Is}\left(X_{r-1}\right)
$$

such that for all points $\left(y_{1}, y_{2}, \ldots, y_{r-1}\right) \in X_{1} \times X_{2} \times \cdots \times X_{r-1}$ there exists $\lambda^{\prime}=\left(\lambda_{1}^{\prime}, \lambda_{2}^{\prime}, \ldots, \lambda_{r-1}^{\prime}\right) \in \Lambda_{r-1}$ such that $\lambda_{i}^{\prime} y_{i} \subset U_{i}\left(h_{i}^{-}\right)$for all $i \in\{1,2, \ldots, r-1\}$. We denote by $\Lambda^{\prime} \subset\langle g, h\rangle^{+}$the finite set of the same words as in $\Lambda_{r-1}$, but now considered as elements in $\operatorname{Is}\left(X_{1}\right) \times \operatorname{Is}\left(X_{2}\right) \times \cdots \times \operatorname{Is}\left(X_{r}\right)$, and fix an arbitrary point $y=\left(y_{1}, y_{2}, \ldots, y_{r}\right) \in X_{1} \times X_{2} \times \cdots \times X_{r}$. By the properties of $\Lambda_{r-1}$ we know that there exists $\lambda^{\prime}=\left(\lambda_{1}^{\prime}, \lambda_{2}^{\prime}, \ldots, \lambda_{r-1}^{\prime}, \lambda_{r}^{\prime}\right) \in \Lambda^{\prime}$ such that

$$
\lambda_{i}^{\prime} y_{i} \subset U_{i}\left(h_{i}^{-}\right) \text {for all } i \in\{1,2, \ldots, r-1\},
$$


but we do not know the position of $\lambda_{r}^{\prime} y_{r} \in X_{r}=U_{r}\left(h_{r}^{+}\right) \cup \bar{X}_{r} \backslash U_{r}\left(h_{r}^{+}\right)$.

However, as in the case $r=1$ the north-south dynamics (9) implies

$$
h_{r}^{-N} g_{r}^{-N} \lambda_{r}^{\prime} y_{r} \in U_{r}\left(h_{r}^{-}\right) \quad \text { or } \quad h_{r}^{-N} \lambda_{r}^{\prime} y_{r} \in U_{r}\left(h_{r}^{-}\right)
$$

according to the two cases $\lambda_{r}^{\prime} y_{r} \in U_{r}\left(h_{r}^{+}\right)$or $\lambda_{r}^{\prime} y_{r} \in \bar{X}_{r} \backslash U_{r}\left(h_{r}^{+}\right)$. Since for $1 \leq i \leq r-1$ we have

$$
h_{i}^{-N} g_{i}^{-N} \cdot U_{i}\left(h_{i}^{-}\right) \subset U_{i}\left(h_{i}^{-}\right) \quad \text { and } \quad h_{i}^{-N} \cdot U_{i}\left(h_{i}^{-}\right) \subset U_{i}\left(h_{i}^{-}\right)
$$

we conclude that the set $\Lambda_{r}$ consisting of all words in $g^{-N}, h^{-N}$ of the form $h^{-N} \lambda^{\prime}$ or $h^{-N} g^{-N} \lambda^{\prime}$ with $\lambda^{\prime} \in \Lambda^{\prime}$ works.

So we have shown the existence of a finite set $\Lambda \subset\langle g, h\rangle$ such that for any $y \in X$ there exists $\lambda=\left(\lambda_{1}, \lambda_{2}, \ldots, \lambda_{r}\right) \in \Lambda$ such that

$$
\lambda^{-1} y \in U_{1}\left(h_{1}^{-}\right) \times U_{2}\left(h_{2}^{-}\right) \times \cdots \times U_{r}\left(h_{r}^{-}\right) .
$$

In particular, by our choice of the neighborhoods $U_{i}\left(h_{i}^{ \pm}\right), i=1,2, \ldots r$, every point $z=\left(z_{1}, z_{2}, \ldots, z_{r}\right) \in U_{1}\left(h_{1}^{+}\right) \times U_{2}\left(h_{2}^{+}\right) \times \cdots \times U_{r}\left(h_{r}^{+}\right) \subset \bar{X}_{1} \times \bar{X}_{2} \times \cdots \times \bar{X}_{r}$ satisfies

$$
d_{i}\left(\sigma_{\lambda_{i}^{-1} y_{i}, z_{i}}, o_{i}\right) \leq c, \quad \text { for all } i \in\{1,2, \ldots, r\} .
$$

We next set $d:=\max \left\{d_{i}\left(o_{i}, \lambda_{i} o_{i}\right): i \in\{1,2, \ldots, r\}, \lambda=\left(\lambda_{1}, \lambda_{2}, \ldots, \lambda_{r}\right) \in \Lambda\right\}$. Then for $i \in\{1,2, \ldots, r\}$ we have

$$
\begin{aligned}
d_{i}\left(\sigma_{y_{i}, \lambda_{i} z_{i}}, o_{i}\right) & \leq d_{i}\left(\lambda_{i} \sigma_{\lambda_{i}^{-1} y_{i}, z_{i}}, \lambda_{i} o_{i}\right)+d_{i}\left(\lambda_{i} o_{i}, o_{i}\right) \\
& <d_{i}\left(\sigma_{\lambda_{i}^{-1} y_{i}, z_{i}}, o_{i}\right)+d \leq c+d .
\end{aligned}
$$

We set $c_{0}:=\sqrt{r}(c+d)$ and $U_{i}:=U_{i}\left(h_{i}^{+}\right) \cap \partial X_{i}$ for $i \in\{1,2, \ldots, r\}$. If $\theta \in E$ and $\tilde{\zeta} \in U_{\theta} \times\{\theta\} \subset \partial X_{\theta}$, then according to (10) its projections $\zeta_{i} \in U_{i}, i \in I^{+}(\theta)$, satisfy

$$
d_{i}\left(\sigma_{y_{i}, \lambda_{i} \zeta_{i}}, o_{i}\right) \leq c+d,
$$

hence by definition (6) of the Weyl chamber with apex $y$ in the class of $\lambda \tilde{\zeta}$ we conclude that for all $t \geq c_{0}$

$$
\mathcal{C}_{y, \lambda \tilde{\zeta}} \cap B_{o}(t) \neq \emptyset, \quad \text { and hence } \quad \lambda \tilde{\zeta} \in \operatorname{Sh}\left(y: B_{o}(t)\right) \cap \partial X_{\theta} .
$$

The claim for $U=U_{1} \times U_{2} \times \cdots \times U_{r} \times E^{+} \subset \partial X^{r e g}$ follows from the fact that

$$
U=\bigcup_{\theta \in E^{+}} U_{\theta}
$$

For $\theta=\left(\theta_{1}, \theta_{2}, \ldots, \theta_{r}\right) \in E=\left\{\theta \in \mathbb{R}_{\geq 0}^{r}:\|\theta\|=1\right\}$ we recall from (4) the definition of the set $\partial X_{\theta} \subset \partial X$ which is homeomorphic to the Cartesian product of the geometric boundaries $\partial X_{i}$ with $i \in I^{+}(\theta)=\left\{i \in\{1,2, \ldots, r\}: \theta_{i}>0\right\}$. The following easy lemma relates the Busemann function (11) of the product to the Busemann functions on the factors. We include a proof for the convenience of the reader. 
LemaA 3.3 Let $\theta=\left(\theta_{1}, \theta_{2}, \ldots, \theta_{r}\right) \in E, x=\left(x_{1}, x_{2}, \ldots, x_{r}\right), y=\left(y_{1}, y_{2}, \ldots, y_{r}\right) \in X$ and $\tilde{\xi} \in \partial X_{\theta}$. If $\xi_{i}$ denotes the projection of $\tilde{\xi}$ to $\partial X_{i}$ then

$$
\beta_{\tilde{\xi}}(x, y)=\sum_{i \in I^{+}(\theta)} \theta_{i} \cdot \beta_{\xi_{i}}\left(x_{i}, y_{i}\right)
$$

Proof. Notice that from the definition of the Busemann functions in $X_{i}, i \in I^{+}(\theta)$, we have

$$
\beta_{\xi_{i}}\left(x_{i}, y_{i}\right)=\lim _{s \rightarrow \infty}\left(s \theta_{i}-d_{i}\left(y_{i}, \sigma_{x_{i}, \xi_{i}}\left(s \theta_{i}\right)\right)\right)
$$

For convenience we set $I^{0}(\theta)=\{1,2, \ldots, r\} \backslash I^{+}(\theta)$. Since $\sum_{i \in I^{+}(\theta)} \theta_{i}^{2}=\sum_{i=1}^{r} \theta_{i}^{2}=1$ we get

$$
\begin{aligned}
\left(s-d\left(y, \sigma_{x, \tilde{\xi}}(s)\right)\right) & \left(s+d\left(y, \sigma_{x, \tilde{\xi}}(s)\right)\right)=s^{2}-d\left(y, \sigma_{x, \tilde{\xi}}(s)\right)^{2} \\
& =s^{2} \sum_{i \in I^{+}(\theta)} \theta_{i}^{2}-\sum_{i \in I^{+}(\theta)} d_{i}\left(y_{i}, \sigma_{x_{i}, \xi_{i}}\left(s \theta_{i}\right)\right)^{2}-\sum_{i \in I^{0}(\theta)} d_{i}\left(y_{i}, x_{i}\right) \\
& =\sum_{i \in I^{+}(\theta)} s^{2} \theta_{i}^{2}-d_{i}\left(y_{i}, \sigma_{x_{i}, \xi_{i}}\left(s \theta_{i}\right)\right)^{2}-\sum_{i \in I^{0}(\theta)} d_{i}\left(y_{i}, x_{i}\right) .
\end{aligned}
$$

So the assertion is proved if we show that for all $i \in I^{+}(\theta)$

$$
\lim _{s \rightarrow \infty} \frac{s \theta_{i}+d_{i}\left(y_{i}, \sigma_{x_{i}, \xi_{i}}\left(s \theta_{i}\right)\right)}{s+d\left(y, \sigma_{x, \tilde{\xi}}(s)\right)}=\theta_{i} ;
$$

this claim follows immediately from the triangle inequalities

$$
\begin{aligned}
& s \theta_{i}-d_{i}\left(y_{i}, x_{i}\right) \leq d_{i}\left(y_{i}, \sigma_{x_{i}, \xi_{i}}\left(s \theta_{i}\right)\right) \leq s \theta_{i}+d_{i}\left(y_{i}, x_{i}\right), \\
& s-d(y, x) \leq d\left(y, \sigma_{x, \tilde{\xi}}(s)\right) \leq s+d(y, x) .
\end{aligned}
$$

To simplify notation in the sequel we further define for $x=\left(x_{1}, x_{2}, \ldots, x_{r}\right)$, $y=\left(y_{1}, y_{2}, \ldots, y_{r}\right) \in X$ and $\tilde{\xi} \in \partial X_{\theta}$ the Busemann vector

$$
\mathcal{B}_{\tilde{\xi}}(x, y)
$$

as the unique vector in $\mathbb{R}^{r}$ with $i$-th coordinate equal to $\beta_{\xi_{i}}\left(x_{i}, y_{i}\right)$ for $i \in I^{+}(\theta)$, and $i$-th coordinate equal to zero for all $i \in I^{0}(\theta)$.

Notice that for $\tilde{\xi} \in \partial X^{r e g}$, the Busemann vector $\mathcal{B}_{\tilde{\xi}}$ is independent of the slope of $\tilde{\xi}$; it only depends on $\left(\xi_{1}, \xi_{2}, \ldots, \xi_{r}\right)=\pi^{F}(\tilde{\xi}) \in \partial^{F} X$. Moreover, by the cocycle identity for the Busemann function we get

$$
\mathcal{B}_{\tilde{\xi}}(x, z)=\mathcal{B}_{\tilde{\xi}}(x, y)+\mathcal{B}_{\tilde{\xi}}(y, z) \quad \text { for all } \quad x, y, z \in X .
$$

We also remark that if $\langle\cdot, \cdot\rangle$ denotes the Euclidean inner product of $\mathbb{R}^{r}$, then the formula in Lemma 3.3 can be rewritten as

$$
\beta_{\tilde{\xi}}(x, y)=\left\langle\mathcal{B}_{\tilde{\xi}}(x, y), \theta\right\rangle .
$$

In the sequel we will also need the following 
Definition 3.4 The directional distance of the ordered pair $(x, y) \in X \times X$ with respect to the slope $\theta \in E$ is defined by

$$
\begin{aligned}
\beta_{\theta}: X \times X & \rightarrow \mathbb{R} \\
(x, y) & \mapsto \beta_{\theta}(x, y) \quad:=\langle H(x, y), \theta\rangle=\sum_{i=1}^{r} \theta_{i} \cdot d_{i}\left(p_{i}(x), p_{i}(y)\right) .
\end{aligned}
$$

In particular, if $\theta \in E$ has $i$-th coordinate 1 and all other coordinates zero then $\beta_{\theta}(x, y)=d_{i}\left(p_{i}(x), p_{i}(y)\right)$.

By $\left(\operatorname{Is}\left(X_{1}\right) \times \operatorname{Is}\left(X_{2}\right) \times \cdots \times \operatorname{Is}\left(X_{r}\right)\right)$-invariance of the distance vector we immediately get that

$$
\beta_{\theta}(g x, g y)=\beta_{\theta}(x, y)
$$

for all $x, y \in X$ and $g \in \operatorname{Is}\left(X_{1}\right) \times \operatorname{Is}\left(X_{2}\right) \times \cdots \times \operatorname{Is}\left(X_{r}\right)$. Moreover, the symmetry and triangle inequality for the distances $d_{1}, d_{2}, \ldots, d_{r}$ directly imply the symmetry and triangle inequality for $\beta_{\theta}$. The following important proposition states that for $\theta \in E^{+}=\left\{\theta \in \mathbb{R}_{>0}^{r}:\|\theta\|=1\right\}$ the directional distance $\beta_{\theta}$ is in fact a distance.

Proposition 3.5 For $\theta \in E^{+}$the directional distance $\beta_{\theta}$ is a distance.

Proof. Let $x=\left(x_{1}, x_{2}, \ldots, x_{r}\right), y=\left(y_{1}, y_{2}, \ldots, y_{r}\right) \in X$. We clearly have

$$
\beta_{\theta}(x, y)=\sum_{i=1}^{r} \theta_{i} \cdot d_{i}\left(x_{i}, y_{i}\right) \geq 0
$$

because all terms involved are non-negative. Moreover, if $\beta_{\theta}(x, y)=0$, then $\theta_{i}>0$ for all $i \in\{1,2, \ldots, r\}$ imply $d_{1}\left(x_{1}, y_{1}\right)=d_{2}\left(x_{2}, y_{2}\right)=\cdots=d_{r}\left(x_{r}, y_{r}\right)=0$, hence $x=y$.

Finally, we have already noticed that the symmetry and triangle inequality follow directly from the symmetry and triangle inequality for the distances $d_{i}$.

The following easy facts will be convenient in the sequel.

Lemma 3.6 Let $x, y \in X$ and $\tilde{\xi} \in \partial X_{\theta}$ for some $\theta \in E$. Then

$$
y \in \mathcal{C}_{x, \tilde{\xi}} \Longleftrightarrow \beta_{\theta}(x, y)=\beta_{\tilde{\xi}}(x, y)
$$

Proof. We write $x=\left(x_{1}, x_{2}, \ldots, x_{r}\right), y=\left(y_{1}, y_{2}, \ldots, y_{r}\right)$ and $\theta=\left(\theta_{1}, \theta_{2}, \ldots, \theta_{r}\right)$. Recall that $I^{+}(\theta)=\left\{i \in\{1,2, \ldots, r\}: \theta_{i}>0\right\}$, and that for $i \in I^{+}(\theta) \xi_{i}$ denotes the projection of $\tilde{\xi}$ to $\partial X_{i}$. Lemma 3.3 and the estimates $\beta_{\xi_{i}}\left(x_{i}, y_{i}\right) \leq d_{i}\left(x_{i}, y_{i}\right)$ imply

$$
\beta_{\tilde{\xi}}(x, y)=\sum_{i \in I^{+}(\theta)} \theta_{i} \cdot \beta_{\xi_{i}}\left(x_{i}, y_{i}\right) \leq \sum_{i=1}^{r} \theta_{i} \cdot d_{i}\left(x_{i}, y_{i}\right)=\langle H(x, y), \theta\rangle=\beta_{\theta}(x, y) .
$$

So we have equality in (13) if and only if for all $i \in I^{+}(\theta)$ the equality

$$
\beta_{\xi_{i}}\left(x_{i}, y_{i}\right)=d_{i}\left(x_{i}, y_{i}\right)
$$


holds; this is precisely the case when $y_{i}$ is a point on the geodesic ray $\sigma_{x_{i} \xi_{i}}$ in $X_{i}$. Therefore - by definition (6) of the Weyl chamber with apex $x$ in the class of $\tilde{\xi}-$ equality in (13) is equivalent to $y \in \mathcal{C}_{x, \tilde{\xi}}$.

If some of the factors $X_{i}$ are geodesically complete, the previous lemma allows to give the following nice geometric interpretation of the directional distance.

Corollary 3.7 Fix $\theta \in E$ and assume that $X_{i}$ is geodesically complete for all $i \in I^{+}(\theta)$. Then for all $x, y \in X$ we have

$$
\beta_{\theta}(x, y)=\max \left\{\beta_{\tilde{\xi}}(x, y): \tilde{\xi} \in \partial X_{\theta}\right\} .
$$

Proof. We first fix $i \in I^{+}(\theta)$. Since $X_{i}$ is geodesically complete, every point $y_{i} \in X_{i} \backslash\left\{x_{i}\right\}$ belongs to a geodesic ray $\sigma_{x_{i}, \xi_{i}}$ with $\xi_{i} \in \partial X_{i}$ the unique extension $\sigma_{x_{i}, y_{i}}(\infty)$ of the geodesic in $X_{i}$ joining $x_{i}$ to $y_{i}$. If $y_{i}=x_{i}$ one may choose an arbitrary point $\xi_{i} \in \partial X_{i}$.

In this way every $y \in X$ determines a (not necessarily unique) boundary point $\tilde{\xi} \in \partial X_{\theta}$ with projections $\xi_{i} \in \partial X_{i}, i \in I^{+}(\theta)$; by choice of $\tilde{\xi}$ and definition (6) we clearly have $y \in \mathcal{C}_{x, \tilde{\xi}}$ and hence, by Lemma 3.6.

$$
\beta_{\theta}(x, y)=\beta_{\tilde{\xi}}(x, y) .
$$

Inequality (13) then proves the claim.

Recall the definition of Weyl chamber shadows from (7) and (8). The following lemma will be needed in the proof of the shadow lemma Theorem 7.2 .

Lemma 3.8 Let $c>0, z=\left(z_{1}, z_{2}, \ldots, z_{r}\right) \in X$ such that $d(o, z)>c, \theta \in E$ and $\tilde{\eta} \in \operatorname{Sh}\left(o: B_{z}(c)\right) \cap \partial X_{\theta}$ with projections $\eta_{i} \in \partial X_{i}, i \in I^{+}(\theta)$. Then we have

$$
0 \leq d_{i}\left(o_{i}, z_{i}\right)-\beta_{\eta_{i}}\left(o_{i}, z_{i}\right)<2 c \quad \text { for all } i \in I^{+}(\theta) .
$$

Proof. By definition $\tilde{\eta} \in \operatorname{Sh}\left(o: B_{z}(c)\right)$ if and only if $\mathcal{C}_{o, \tilde{\eta}} \cap B_{z}(c) \neq \emptyset$. Hence if $\tilde{\eta} \in \partial X_{\theta}$ then for all $i \in I^{+}(\theta)$ there exists $t_{i} \geq 0$ such that $y_{i}:=\sigma_{o_{i}, \eta_{i}}\left(t_{i}\right) \in p_{i}\left(B_{z}(c)\right)$. Necessarily we have

$$
d_{i}\left(z_{i}, \sigma_{o_{i}, \eta_{i}}\right) \leq d_{i}\left(z_{i}, y_{i}\right)<c \quad \text { for all } i \in I^{+}(\theta),
$$

hence the claim follows from Lemma 2.1.

\section{The exponent of growth}

For the remainder of the article $X$ will be a product of locally compact Hadamard spaces $X_{1}, X_{2}, \ldots, X_{r}$, and $\Gamma<\operatorname{Is}\left(X_{1}\right) \times \operatorname{Is}\left(X_{2}\right) \times \cdots \times \operatorname{Is}\left(X_{r}\right)$ a group acting properly discontinuously by isometries on $X$ which contains two elements $h=\left(h_{1}, h_{2}, \ldots, h_{r}\right)$ and $g=\left(g_{1}, g_{2}, \ldots, g_{r}\right)$ such that for $i \in\{1,2, \ldots, r\} g_{i}$ and $h_{i}$ are independent rank one isometries of $X_{i}$. We further fix a base point $o=\left(o_{1}, o_{2}, \ldots, o_{r}\right) \in \operatorname{Ax}(h)$. 
We recall that the geometric limit set of $\Gamma$ is defined by $L_{\Gamma}:=\overline{\Gamma \cdot x} \cap \partial X$, where $x \in X$ is arbitrary. In this section we recall the notion of exponent of growth introduced in [Lin10] and [Lin13] and give an important criterion for divergence or convergence of certain sums over $\Gamma$. This will play a central role in the construction of (generalized) conformal densities in Sections 5 and 6.

We recall the notation introduced in Section 3 , in particular, we denote $E \subset \mathbb{R}^{r}$ the set of unit vectors in $\mathbb{R}_{\geq 0}^{r}$. For $x, y \in X, \theta \in E$ and $\varepsilon>0$ we first set

$$
\Gamma(x, y ; \theta, \varepsilon):=\{\gamma \in \Gamma: \gamma y \neq x \quad \text { and }\|\widehat{H}(x, \gamma y)-\theta\|<\varepsilon\} .
$$

In order to define the exponent of growth of $\Gamma$ of slope $\theta$ we set

$$
\delta_{\theta}^{\varepsilon}(x, y):=\inf \left\{s>0: \sum_{\gamma \in \Gamma(x, y ; \theta, \varepsilon)} \mathrm{e}^{-s d(x, \gamma y)} \text { converges }\right\} .
$$

If $\delta(\Gamma)$ denotes the critical exponent of $\Gamma$ defined by

$$
\delta(\Gamma):=\inf \left\{s>0: \sum_{\gamma \in \Gamma} \mathrm{e}^{-s d(o, \gamma o)} \text { converges }\right\},
$$

we clearly have $\delta_{\theta}^{\varepsilon}(x, y) \leq \delta(\Gamma)$ with equality if $\varepsilon>\sqrt{2}$. Moreover, an easy calculation shows that $\delta_{\theta}^{\varepsilon}(x, y)$ is related to the numbers

$$
\Delta N_{\theta}^{\varepsilon}(x, y ; n):=\#\{\gamma \in \Gamma: n-1 \leq d(x, \gamma y)<n,\|\widehat{H}(x, \gamma y)-\theta\|<\varepsilon\}
$$

with $n \in \mathbb{N}, n \geq 2$ via

$$
\delta_{\theta}^{\varepsilon}(x, y)=\limsup _{n \rightarrow \infty} \frac{\ln \Delta N_{\theta}^{\varepsilon}(x, y ; n)}{n} .
$$

Recall that the exponent of growth of $\Gamma$ of slope $\theta$ is defined by

$$
\delta_{\theta}(\Gamma):=\lim _{\varepsilon \rightarrow 0} \delta_{\theta}^{\varepsilon}(o, o) .
$$

Notice that this number $\delta_{\theta}(\Gamma)$ does not depend on the choice of arguments of $\delta_{\theta}^{\varepsilon}$ by elementary geometric estimates; it can be interpreted as an exponential growth rate of the number of orbit points which are "close" to a geodesic ray in the class of a boundary point with slope $\theta$. Moreover, we clearly have $\delta_{\theta}(\Gamma) \leq \delta(\Gamma)$ for all $\theta \in E$.

Furthermore, we recall the following properties from Section 7 in [Lin13:

\section{Properties:}

(a) $L_{\Gamma} \cap \partial X_{\theta} \neq \emptyset$ if and only if $\delta_{\theta}(\Gamma) \geq 0$.

(b) The map $E \rightarrow \mathbb{R}, \hat{\theta} \mapsto \delta_{\hat{\theta}}(\Gamma)$ is upper semi-continuous.

It will turn out useful to consider the homogeneous extension $\Psi_{\Gamma}: \mathbb{R}_{\geq 0}^{r} \rightarrow \mathbb{R}$ of the map $E \rightarrow \mathbb{R}, \hat{\theta} \mapsto \delta_{\hat{\theta}}(\Gamma)$. Theorem 7.6 in [Lin13] states that $\Psi_{\Gamma}$ is concave. This implies in particular that there exists a unique $\theta^{*} \in E$ such that $\delta_{\theta^{*}}(\Gamma)=\max \left\{\delta_{\theta}(\Gamma): \theta \in E\right\}$. The following important proposition will play a key role in the proof of Theorem A and for the construction of generalized conformal densities. Recall definitions (2) and (3) for the distance vector and the direction of a pair of points in $X$. 
Proposition 4.1 Let $f: \mathbb{R}_{\geq 0}^{r} \rightarrow \mathbb{R}$ be a continuous homogeneous function, $D \subset E$ a a relatively open set and put $\Gamma_{D}:=\{\gamma \in \Gamma: \gamma o \neq o, \widehat{H}(o, \gamma o) \in D\}$.

(a) If there exists $\hat{\theta} \in D$ such that $f(\hat{\theta})<\delta_{\hat{\theta}}(\Gamma)$, then the sum $\sum_{\gamma \in \Gamma_{D}} \mathrm{e}^{-f(H(o, \gamma o))}$ diverges.

(b) If $f(\theta)>\delta_{\theta}(\Gamma)$ for all $\theta \in \bar{D}$, then the sum $\sum_{\gamma \in \Gamma_{D}} \mathrm{e}^{-f(H(o, \gamma o))}$ converges.

Proof. For $\gamma \in \Gamma$ we abbreviate $\theta_{\gamma}:=\widehat{H}(o, \gamma o)=\frac{H(o, \gamma o)}{d(o, \gamma o)}$.

(a) Let $\hat{\theta} \in D$ such that $f(\hat{\theta})<\delta_{\hat{\theta}}(\Gamma)$. Since $\delta_{\hat{\theta}}(\Gamma)=\lim _{\varepsilon \rightarrow 0} \delta_{\hat{\theta}}^{\varepsilon}(o, o)$, there exists $\varepsilon>0$ and $\hat{s} \in \mathbb{R}$ such that for $\gamma \in \Gamma_{D}$ with $\left\|\theta_{\gamma}-\hat{\theta}\right\|<\varepsilon$ we have

$$
f\left(\theta_{\gamma}\right)<\hat{s}<\delta_{\hat{\theta}}^{\varepsilon}(o, o) .
$$

Since $f(H(o, \gamma o))=f\left(\theta_{\gamma}\right) \cdot d(o, \gamma o)$ we estimate

$$
\sum_{\gamma \in \Gamma_{D}} \mathrm{e}^{-f(H(o, \gamma o))}>\sum_{\gamma \in \Gamma(o, o ; \hat{\theta}, \varepsilon)} \mathrm{e}^{-\hat{s} d(o, \gamma o)},
$$

and the latter sum diverges because $\hat{s}<\delta_{\hat{\theta}}^{\varepsilon}(o, o)$.

(b) Let $\hat{\theta} \in \bar{D}$. Since $f(\hat{\theta})>\delta_{\hat{\theta}}(\Gamma)=\lim _{\varepsilon \rightarrow 0} \delta_{\hat{\theta}}^{\varepsilon}(o, o)$, there exists $\varepsilon^{\prime}>0$ and $\hat{s}<f(\hat{\theta})$ such that

$$
\delta_{\hat{\theta}}^{\varepsilon^{\prime}}(o, o)<\hat{s}<f(\hat{\theta})
$$

For $\theta \in E$ and $\varepsilon>0$ we set $B_{\theta}(\varepsilon):=\left\{\theta^{\prime} \in E:\left\|\theta^{\prime}-\theta\right\|<\varepsilon\right\}$. The continuity of the function $f$ and inequality (16) imply the existence of $\hat{\varepsilon}<\varepsilon^{\prime}$ such that for any $\theta \in B_{\hat{\theta}}(\hat{\varepsilon})$ we have $\hat{s}<f(\theta)$. Hence for all $z \in X$ with $\theta_{z}:=\widehat{H}(o, z) \in B_{\hat{\theta}}(\hat{\varepsilon})$ we have

$$
f\left(\theta_{z}\right)=\frac{f(H(o, z))}{d(o, z)}>\hat{s}>\delta_{\hat{\theta}}^{\varepsilon^{\prime}}(o, o) \geq \delta_{\hat{\theta}}^{\hat{\varepsilon}}(o, o) .
$$

We now choose a sequence $\left(\theta_{j}\right) \subset \bar{D}$ and corresponding sequences $\left(\varepsilon_{j}\right) \subset \mathbb{R}_{>0}$ and $\left(s_{j}\right) \subset \mathbb{R}_{>0}$ such that for every $\theta \in B_{\theta_{j}}\left(\varepsilon_{j}\right)$ we have

$$
\delta_{\theta_{j}}^{\varepsilon_{j}}(o, o)<s_{j}<f(\theta)
$$

and such that the sets $B_{\theta_{j}}\left(\varepsilon_{j}\right), j \in \mathbb{N}$, cover $\bar{D}$. By compactness of $\bar{D}$ there exists a finite set $J \subset \mathbb{N}$ with $\bar{D} \subset \bigcup_{j \in J} B_{\theta_{j}}\left(\varepsilon_{j}\right)$, and we conclude

$$
\begin{aligned}
\sum_{\gamma \in \Gamma_{D}} \mathrm{e}^{-f(H(o, \gamma o))} & \leq \sum_{j \in J} \sum_{\gamma \in \Gamma\left(o, o ; \theta_{j}, \varepsilon_{j}\right)} \mathrm{e}^{-f(H(o, \gamma o))} \\
& \leq \sum_{j \in J} \sum_{\gamma \in \Gamma\left(o, o ; \theta_{j}, \varepsilon_{j}\right)} \mathrm{e}^{-s_{j} d(o, \gamma o)}<\infty
\end{aligned}
$$


because $s_{j}>\delta_{\theta_{j}}^{\varepsilon_{j}}(o, o)$ for $j \in J$.

Taking $D=E$ and $f(H)=s \cdot\|H\|$ we obtain as a corollary that

$$
\delta(\Gamma)=\max \left\{\delta_{\theta}(\Gamma): \theta \in E\right\}=\delta_{\theta^{*}}(\Gamma) .
$$

We conclude this section with two illustrative examples.

Example 1 (see [Lin13, Section 7]) We let $X=X_{1} \times X_{2} \times \cdots \times X_{r}$ be a product of Hadamard manifolds with pinched negative curvature, and assume that for all $i \in\{1,2, \ldots, r\}$ a discrete convex cocompact group $\Gamma_{i}<\operatorname{Is}\left(X_{i}\right)$ with critical exponent $\delta_{i}>0$ is given. Then the exponent of growth of slope $\theta \in E$ for the product group $\Gamma:=\Gamma_{1} \times \Gamma_{2} \times \cdots \times \Gamma_{r}$ satisfies

$$
\delta_{\theta}(\Gamma)=\sum_{i=1}^{r} \delta_{i} \theta_{i}
$$

Using Lagrange multipliers one can easily show that this number is maximal for $\theta^{*} \in E^{+}$with coordinates

$$
\theta_{i}^{*}=\frac{\delta_{i}}{\delta_{1}^{2}+\delta_{2}^{2}+\cdots+\delta_{r}^{2}}, \quad i \in\{1,2, \ldots, r\}
$$

in particular we have

$$
\delta(\Gamma)=\delta_{\theta^{*}}(\Gamma)=\sqrt{\delta_{1}^{2}+\delta_{2}^{2}+\cdots+\delta_{r}^{2}} .
$$

The homogeneous function $\Psi_{\Gamma}: \mathbb{R}_{>0}^{r} \rightarrow \mathbb{R}$ is simply the linear functional defined by taking the inner product $\langle\cdot, \cdot\rangle$ in $\mathbb{R}^{r}$ with the unique vector in $\mathbb{R}_{>0}^{r}$ with coordinates $\delta_{i}$.

ExAmple 2 Consider a product of hyperbolic planes $X=H^{2} \times H^{2}$ and a Hilbert modular group $\Gamma<\operatorname{Is}(X)$. Then $\Gamma$ is an irreducible non-uniform lattice in a higher rank symmetric space, hence from Proposition 7.2 and 7.3 in Alb99. we know that

$$
\Psi_{\Gamma}=\left\langle\left(\begin{array}{c}
1 \\
1
\end{array}\right), \cdot\right\rangle, \quad \text { so } \quad \delta_{\theta}(\Gamma)=\theta_{1}+\theta_{2}
$$

Here $\delta_{\theta}(\Gamma)$ is maximal for

$$
\theta^{*}=\frac{1}{\sqrt{2}}\left(\begin{array}{c}
1 \\
1
\end{array}\right) \in E^{+},
$$

so we get $\delta(\Gamma)=\delta_{\theta^{*}}(\Gamma)=\sqrt{2}$.

\section{The classical Patterson-Sullivan construction}

In this section we will construct a conformal density for $\Gamma$ using an idea originally due to S. J. Patterson ( [Pat76]) in the context of Fuchsian groups. Taking advantage 
of Proposition 4.1 we will be able to describe precisely its support and hence prove Theorem A.

Recall that a $\Gamma$-invariant conformal density of dimension $\delta \geq 0$ is a map $\mu$ from $X$ to the cone $\mathcal{M}^{+}(\partial X)$ of positive finite Borel measures on $\partial X$ such that $\operatorname{supp}\left(\mu_{o}\right) \subset L_{\Gamma}$, $\gamma_{*} \mu_{x}=\mu_{\gamma x}$ for all $\gamma \in \Gamma, x \in X$ and

$$
\frac{d \mu_{x}}{d \mu_{o}}(\tilde{\eta})=\mathrm{e}^{\delta \beta_{\tilde{\eta}}(o, x)} \quad \text { for all } \tilde{\eta} \in \operatorname{supp}\left(\mu_{o}\right), x \in X .
$$

In order to construct a $\Gamma$-invariant conformal density of dimension $\delta(\Gamma)$ we first suppose that we are given a map $b: \Gamma \rightarrow \mathbb{R}, \gamma \mapsto b_{\gamma}$ such that the sum

$$
\sum_{\gamma \in \Gamma} \mathrm{e}^{-s b_{\gamma}}
$$

has exponent of convergence $s=1$ (which means that it converges for $s>1$ and diverges for $s<1$ ). The following useful lemma states that if the above sum converges for $s=1$, then we can slightly modify it to obtain a sum which diverges for $s \leq 1$ and converges for $s>1$.

Lemma 5.1 ( (PATterson [PAT76])) If the sum [17) has exponent of convergence $s=1$, then there exists a non-decreasing continuous function $h:[0, \infty) \rightarrow[1, \infty)$ such that

(i) $\sum_{\gamma \in \Gamma} \mathrm{e}^{-s b_{\gamma}} h\left(\mathrm{e}^{b_{\gamma}}\right)$ has exponent of convergence $s=1$ and diverges at $s=1$;

(ii) for any $\alpha>0$ there exists $r_{0}>0$ such that for $r \geq r_{0}$ and $t>1$

$$
h(r t) \leq t^{\alpha} h(r) .
$$

Notice that if the sum (17) already diverges at $s=1$, then $h$ can be chosen as the constant function identical to 1.

Recall the definition of the exponent of growth of $\Gamma$ and its properties from Section 4. We have already noticed that there exists a unique unit vector $\theta^{*} \in E$ such that $\delta(\Gamma)=\delta_{\theta^{*}}(\Gamma)$.

Following the original idea of Patterson [Pat76], we apply the above lemma to the map

$$
b: \Gamma \rightarrow \mathbb{R}, \quad \gamma \mapsto \delta(\Gamma) \cdot d(o, \gamma o)
$$

Then by definition (14) of the critical exponent $\delta(\Gamma)$ the sum

$$
\sum_{\gamma \in \Gamma} \mathrm{e}^{-s b_{\gamma}}=\sum_{\gamma \in \Gamma} \mathrm{e}^{-s \delta(\Gamma) \cdot d(o, \gamma o)}
$$

has exponent of convergence $s=1$. Let $h:[0, \infty) \rightarrow[1, \infty)$ be a non-decreasing function as in Patterson's Lemma above and define

$$
P^{s}:=\sum_{\gamma \in \Gamma} \mathrm{e}^{-s b_{\gamma}} h\left(\mathrm{e}^{b_{\gamma}}\right)
$$


If $D$ denotes the unit Dirac point measure, then for $s>1$ we get a probability measure on $X$ by setting

$$
\mu_{o}^{s}:=\frac{1}{P^{s}} \sum_{\gamma \in \Gamma} \mathrm{e}^{-s b_{\gamma}} h\left(\mathrm{e}^{b_{\gamma}}\right) D(\gamma o) .
$$

Notice that by construction any weak accumulation point $\mu_{o}$ of $\mu_{o}^{s}$ as $s \searrow 1$ is a probability measure on $\partial X$ with $\operatorname{supp}\left(\mu_{o}\right) \subset L_{\Gamma}$.

Before we continue with the construction of a $\Gamma$-invariant conformal density we state an auxiliary lemma which will be useful in the sequel. For a topological space $Y$ we denote $\left(\mathrm{C}^{0}(Y),\|\cdot\|_{\infty}\right)$ the space of real valued continuous functions on $Y$ with norm $\|f\|_{\infty}=\sup \{|f(y)|: y \in Y\}, f \in \mathrm{C}^{0}(Y)$.

Lemma 5.2 Fix $x, y \in X$ and $s>0$. Let $h:[0, \infty) \rightarrow[1, \infty)$ be a non-decreasing function as in Patterson's Lemma 5.1, and $b: X \times X \rightarrow \mathbb{R}$ a continuous map with the property

$$
|b(x, z)-b(y, z)| \leq C \cdot d(x, y) \quad \text { for all } \quad z \in X .
$$

Then the continuous function

$$
g_{x, y}^{s}: X \rightarrow \mathbb{R}, \quad z \mapsto \frac{\mathrm{e}^{-s b(x, z)} h\left(\mathrm{e}^{b(x, z)}\right)}{\mathrm{e}^{-s b(y, z)} h\left(\mathrm{e}^{b(y, z)}\right)}
$$

satisfies

$$
\lim _{s \rightarrow 1}\left\|g_{x, y}^{s}-g_{x, y}^{1}\right\|_{\infty}=0
$$

Proof. We first remark that for all $z \in X$ we have

$$
\frac{g_{x, y}^{s}(z)}{g_{x, y}^{1}(z)}=\frac{\mathrm{e}^{s(b(y, z)-b(x, z))}}{\mathrm{e}^{b(y, z)-b(x, z)}}=\mathrm{e}^{(s-1)(b(y, z)-b(x, z))},
$$

so by the property (21) of $b$ we get

$$
\mathrm{e}^{-|s-1| C \cdot d(x, y)} \leq \frac{g_{x, y}^{s}(z)}{g_{x, y}^{1}(z)} \leq \mathrm{e}^{|s-1| C \cdot d(x, y)}
$$

Using the inequality $\mathrm{e}^{t}+\mathrm{e}^{-t} \geq 2$ we obtain for all $z \in X$

$$
\left|\frac{g_{x, y}^{s}(z)}{g_{x, y}^{1}(z)}-1\right| \leq \mathrm{e}^{|s-1| C \cdot d(x, y)}-1 .
$$

By Patterson's Lemma (ii) there exists $r_{0}>0$ such that for all $r \geq r_{0}$ and $t>1$ we have

$$
h(r t) \leq t^{2} h(r) \leq \mathrm{e}^{2 t} h(r) .
$$

So for $z \in X$ such that $b(y, z) \geq \ln \left(r_{0}\right)$ we get

$$
\frac{h\left(\mathrm{e}^{b(x, z)}\right)}{h\left(\mathrm{e}^{b(y, z)}\right)} \leq \mathrm{e}^{2(b(x, z)-b(y, z))} \leq \mathrm{e}^{2 C \cdot d(x, y)}
$$


If $z \in X$ satisfies $b(y, z)<\ln \left(r_{0}\right)$, then

$$
b(x, z)=(b(x, z)-b(y, z))+b(y, z) \leq C \cdot d(x, y)+\ln \left(r_{0}\right)
$$

hence since $h$ is a non-decreasing function $\geq 1$

$$
\frac{h\left(\mathrm{e}^{b(x, z)}\right)}{h\left(\mathrm{e}^{b(y, z)}\right)} \leq h\left(\mathrm{e}^{b(x, z)}\right) \leq h\left(\mathrm{e}^{C \cdot d(x, y)} r_{0}\right) .
$$

This implies that there exists a constant $K>1$ (which only depends on $d(x, y)$ ) such that for all $z \in X$

$$
\frac{h\left(\mathrm{e}^{b(x, z)}\right)}{h\left(\mathrm{e}^{b(y, z)}\right)} \leq K
$$

We conclude

$$
\begin{aligned}
\left|g_{x, y}^{s}(z)-g_{x, y}^{1}(z)\right| & =g_{x, y}^{1}(z) \cdot\left|\frac{g_{x, y}^{s}(z)}{g_{x, y}^{1}(z)}-1\right| \leq \frac{\mathrm{e}^{-b(x, z)} h\left(\mathrm{e}^{b(x, z)}\right)}{\mathrm{e}^{-b(y, z)} h\left(\mathrm{e}^{b(y, z)}\right)}\left(\mathrm{e}^{|s-1| C \cdot d(x, y)}-1\right) \\
& \leq \mathrm{e}^{C \cdot d(x, y)} \cdot K\left(\mathrm{e}^{|s-1| C \cdot d(x, y)}-1\right)
\end{aligned}
$$

so

$$
\left\|g_{x, y}^{s}-g_{x, y}^{1}\right\|_{\infty}=\sup _{z \in X}\left|g_{x, y}^{s}(z)-g_{x, y}^{1}(z)\right| \longrightarrow 0 \quad \text { as } \quad s \rightarrow 1
$$

In order to obtain a $\Gamma$-invariant conformal density, we imitate the construction (20) and define for $x, z \in X$ and $\gamma \in \Gamma$

$$
b(x, z):=\delta(\Gamma) \cdot d(x, z), \quad b_{\gamma}:=b(o, \gamma o) .
$$

Notice that by the triangle inequality for the distance function the map $b: X \times X \rightarrow \mathbb{R}$ is continuous and satisfies property (21) with $C=\delta(\Gamma)$.

For $s>1, x \in X$ and with $P^{s}$ as defined in (19) we get a family of positive finite Borel measures on $\bar{X}$ via

$$
\mu_{x}^{s}:=\frac{1}{P^{s}} \sum_{\gamma \in \Gamma} \mathrm{e}^{-s b(x, \gamma o)} h\left(\mathrm{e}^{b(x, \gamma o)}\right) D(\gamma o)
$$

in particular, $\mu_{o}^{s}$ is precisely the probability measure defined in (20). For fixed $s>1$ the measures $\mu_{x}^{s}, x \in X$, are $\Gamma$-equivariant by construction and absolutely continuous with respect to each other with Radon Nikodym derivative

$$
\frac{\mathrm{d} \mu_{x}^{s}}{\mathrm{~d} \mu_{y}^{s}}: \operatorname{supp}\left(\mu_{y}^{s}\right) \rightarrow \mathbb{R}, \quad z \mapsto \frac{\mathrm{e}^{-s b(x, z)} h\left(\mathrm{e}^{b(x, z)}\right)}{\mathrm{e}^{-s b(y, z)} h\left(\mathrm{e}^{b(y, z)}\right)}=g_{x, y}^{s}(z),
$$

where $g_{x, y}^{s}$ is the continuous function defined by (22) in Lemma 5.2 ,

Moreover, we have the following

Lemma 5.3 For fixed $x, y \in X$ and $s>0$ the continuous function

$$
g_{x, y}^{s}: X \rightarrow \mathbb{R}, \quad z \mapsto \frac{\mathrm{e}^{-s b(x, z)} h\left(\mathrm{e}^{b(x, z)}\right)}{\mathrm{e}^{-s b(y, z)} h\left(\mathrm{e}^{b(y, z)}\right)}
$$


extends continuously to $\bar{X}$. Moreover, if $\left(z_{n}\right) \subset X$ is a sequence converging to $\tilde{\eta} \in \partial X$, then

$$
\lim _{n \rightarrow \infty} g_{x, y}^{s}\left(z_{n}\right)=\mathrm{e}^{-s \delta(\Gamma) \beta_{\tilde{\eta}}(x, y)}=\mathrm{e}^{s \delta(\Gamma) \beta_{\tilde{\eta}}(y, x)} .
$$

Proof. We first notice that if $\left(z_{n}\right) \subset X$ is a sequence converging to a point $\tilde{\eta} \in \partial X$, then the map

$$
d\left(x, z_{n}\right)-d\left(\cdot, z_{n}\right) \quad \text { converges to the map } \beta_{\tilde{\eta}}(x, \cdot)
$$

uniformly on compact sets. So

$$
\frac{\mathrm{e}^{-s b\left(x, z_{n}\right)}}{\mathrm{e}^{-s b\left(y, z_{n}\right)}}=\mathrm{e}^{-s\left(b\left(x, z_{n}\right)-b\left(y, z_{n}\right)\right)}=\mathrm{e}^{-s \delta(\Gamma)\left(d\left(x, z_{n}\right)-d\left(y, z_{n}\right)\right)} \longrightarrow \mathrm{e}^{-s \delta(\Gamma) \beta_{\tilde{\eta}}(x, y)}
$$

as $n \rightarrow \infty$. Hence it suffices to prove that for any sequence $\left(z_{n}\right) \subset X$ with $d\left(o, z_{n}\right) \rightarrow \infty$ we have

$$
\lim _{n \rightarrow \infty} \frac{h\left(\mathrm{e}^{b\left(x, z_{n}\right)}\right)}{h\left(\mathrm{e}^{b\left(y, z_{n}\right)}\right)}=1
$$

Let $\varepsilon>0$ be arbitrary and fix $\alpha<\frac{\ln (1+\varepsilon)}{\delta(\Gamma) d(x, y)}$. Then by Patterson's Lemma 5.1 (ii) there exists $r_{0}>0$ such that for $r \geq r_{0}$ and $t>1$

$$
\frac{h(r t)}{h(r)} \leq t^{\alpha}
$$

So for all $z \in X$ with $b(x, z) \geq \ln \left(r_{0}\right)$ and $b(y, z) \geq \ln \left(r_{0}\right)$ we get

$$
\mathrm{e}^{-\alpha|b(x, z)-b(y, z)|} \leq \frac{h\left(\mathrm{e}^{b(x, z)}\right)}{h\left(\mathrm{e}^{b(y, z)}\right)} \leq \mathrm{e}^{\alpha|b(x, z)-b(y, z)|} .
$$

By the remark following the definition of $b$ we have $|b(x, z)-b(y, z)| \leq \delta(\Gamma) d(x, y)$ for all $z \in X$, hence by choice of $\alpha$

$$
\mathrm{e}^{-\alpha \delta(\Gamma) d(x, y)} \leq \frac{h\left(\mathrm{e}^{b(x, z)}\right)}{h\left(\mathrm{e}^{b(y, z)}\right)} \leq \mathrm{e}^{\alpha \delta(\Gamma) d(x, y)}<1+\varepsilon .
$$

Using again the inequality $\mathrm{e}^{t}+\mathrm{e}^{-t} \geq 2$ we obtain as a lower bound

$$
\frac{h\left(\mathrm{e}^{b(x, z)}\right)}{h\left(\mathrm{e}^{b(y, z)}\right)} \geq \mathrm{e}^{-\alpha \delta(\Gamma) d(x, y)} \geq 2-\mathrm{e}^{\alpha \delta(\Gamma) d(x, y)}>2-(1+\varepsilon)=1-\varepsilon .
$$

Hence for all $n \in \mathbb{N}$ such that $d\left(o, z_{n}\right) \geq \frac{\ln \left(r_{0}\right)}{\delta(\Gamma)}+\max \{d(o, x), d(o, y)\}$ we have

$$
\left|1-\frac{h\left(\mathrm{e}^{b\left(x, z_{n}\right)}\right)}{h\left(\mathrm{e}^{b\left(y, z_{n}\right)}\right)}\right|<\varepsilon
$$

Recall that $\mathcal{M}^{+}(\partial X)$ denotes the cone of positive finite Borel measures on $\partial X$. 
Proposition 5.4 Let $\left(s_{j}\right) \subset \mathbb{R}, s_{j} \searrow 1$ be a sequence such that $\mu_{o}^{s_{j}}$ converges weakly to $\mu_{o}$, and $x \in X$ arbitrary. Then the sequence of measures $\mu_{x}^{s_{j}}$ converges weakly to a measure $\mu_{x} \in \mathcal{M}^{+}(\partial X)$ with $\operatorname{supp}\left(\mu_{x}\right) \subset L_{\Gamma}$ and

$$
\frac{d \mu_{x}}{d \mu_{o}}(\tilde{\eta})=\mathrm{e}^{\delta(\Gamma) \beta_{\tilde{\eta}}(o, x)} \quad \text { for all } \tilde{\eta} \in \operatorname{supp}\left(\mu_{o}\right) .
$$

Proof. Let $f \in \mathrm{C}^{0}(\bar{X})$ with $\|f\|_{\infty}<\infty$ be arbitrary, $s>1$ and denote $g_{x, o}^{s}: X \rightarrow \mathbb{R}$ the function defined by (22) in Lemma 5.2 (which extends continuously to $\bar{X}$ by Lemma 5.3). By (23) we have for all $s>1$ and for all $z \in \operatorname{supp}\left(\mu_{o}^{s}\right)$

$$
\frac{\mathrm{d} \mu_{x}^{s}}{\mathrm{~d} \mu_{o}^{s}}(z)=\frac{\mathrm{e}^{-s b(x, z)} h\left(\mathrm{e}^{b(x, z)}\right)}{\mathrm{e}^{-s b(o, z)} h\left(\mathrm{e}^{b(o, z)}\right)}=g_{x, o}^{s}(z),
$$

hence

$$
\int_{\bar{X}} f(z) \mathrm{d} \mu_{x}^{s}(z)=\int_{\bar{X}} f(z) g_{x, o}^{s}(z) \mathrm{d} \mu_{o}^{s}(z)
$$

We claim that for any sequence $\left(s_{j}\right) \searrow 1$ such that $\mu_{o}^{s_{j}}$ converges weakly to $\mu_{o}$ we have

$$
\lim _{j \rightarrow \infty} \int_{\bar{X}} f(z) \mathrm{d} \mu_{x}^{s_{j}}(z)=\int_{\bar{X}} f(\tilde{\eta}) \mathrm{e}^{\delta(\Gamma) \beta_{\tilde{\eta}}(o, x)} \mathrm{d} \mu_{o}(\tilde{\eta}) ;
$$

so the measure $\mu_{x}$ defined by

$$
\frac{\mathrm{d} \mu_{x}}{\mathrm{~d} \mu_{o}}(\tilde{\eta})=\mathrm{e}^{\delta(\Gamma) \beta_{\tilde{\eta}}(o, x)} \quad \text { for all } \tilde{\eta} \in \operatorname{supp}\left(\mu_{o}\right)
$$

is the weak limit of the sequence of measures $\mu_{x}^{s_{j}}$. Hence in particular we have

$$
\operatorname{supp}\left(\mu_{x}\right) \subset \operatorname{supp}\left(\mu_{o}\right) \subset L_{\Gamma} .
$$

In order to prove $(*)$ we notice that by Lemma 5.3 we have for $\tilde{\eta} \in \operatorname{supp}\left(\mu_{o}\right) \subset \partial X$

$$
\mathrm{e}^{\delta(\Gamma) \beta_{\tilde{\eta}}(o, x)}=g_{x, o}^{1}(\tilde{\eta})
$$

we estimate

$$
\begin{aligned}
\mid \int_{\bar{X}} f(z) \mathrm{d} \mu_{x}^{s_{j}}(z) & -\int_{\bar{X}} f(\tilde{\eta}) \mathrm{e}^{\delta(\Gamma) \beta_{\tilde{\eta}}(o, x)} \mathrm{d} \mu_{o}(\tilde{\eta}) \mid \\
& \leq\left|\int_{\bar{X}} f(z) g_{x, o}^{s_{j}}(z) \mathrm{d} \mu_{o}^{s_{j}}(z)-\int_{\bar{X}} f(z) g_{x, o}^{1}(z) \mathrm{d} \mu_{o}^{s_{j}}(z)\right| \\
& +\left|\int_{\bar{X}} f(z) g_{x, o}^{1}(z) \mathrm{d} \mu_{o}^{s_{j}}(z)-\int_{\bar{X}} f(\tilde{\eta}) g_{x, o}^{1}(\tilde{\eta}) \mathrm{d} \mu_{o}(\tilde{\eta})\right| .
\end{aligned}
$$

Since $f \cdot g_{x, o}^{1}$ is a bounded and continuous function on $\bar{X}$, and $\mu_{o}^{s_{j}}$ converges weakly to $\mu_{o}$, the second term tends to zero as $j$ tends to infinity. For the first term we get by 
definition of the measure $\mu_{o}^{s_{j}}$

$$
\begin{aligned}
\mid \int_{\bar{X}} f(z) g_{x, o}^{s_{j}}(z) \mathrm{d} \mu_{o}^{s_{j}}(z) & -\int_{\bar{X}} f(z) g_{x, o}^{1}(z) \mathrm{d} \mu_{o}^{s_{j}}(z) \mid \\
& =\left|\int_{\bar{X}} f(z)\left(g_{x, o}^{s_{j}}(z)-g_{x, o}^{1}(z)\right) \mathrm{d} \mu_{o}^{s_{j}}(z)\right| \\
& =\frac{1}{P^{s_{j}}} \sum_{\gamma \in \Gamma} f(\gamma o)\left(g_{x, o}^{s_{j}}(\gamma o)-g_{x, o}^{1}(\gamma o)\right) \mathrm{e}^{-s_{j} b(o, \gamma o)} h\left(\mathrm{e}^{b(o, \gamma o)}\right) \\
& \leq\|f\|_{\infty}\left\|g_{x, o}^{s_{j}}-g_{x, o}^{1}\right\|_{\infty} \longrightarrow 0
\end{aligned}
$$

as $j \rightarrow \infty$ by Lemma 5.2 .

Recall that $\theta^{*} \in E$ is the unique unit vector such that $\delta_{\theta^{*}}(\Gamma)=\delta(\Gamma)$. In order to prove Theorem A it remains to show that the support of the conformal density $\mu$ constructed above is included in the unique $\Gamma$-invariant subset of the limit set which consists of all limit points with slope $\theta^{*}$. For that we need the following auxiliary result which easily follows from Proposition 4.1 (b).

Lemma 5.5 If $b_{\gamma}$ is given by (18) and $h$ is a non-decreasing function as in Patterson's Lemma 5.1, then for all $\varepsilon>0$

$$
\sum_{\substack{\gamma \in \Gamma \\\left\|\widehat{H}(o, \gamma o)-\theta^{*}\right\|>\varepsilon}} \mathrm{e}^{-b_{\gamma}} h\left(\mathrm{e}^{b_{\gamma}}\right)<\infty .
$$

Proof. Let $\varepsilon>0$ arbitrary and set

$$
s_{\varepsilon}:=\max \left\{\delta_{\theta}(\Gamma): \theta \in E,\left\|\theta-\theta^{*}\right\| \geq \varepsilon\right\} .
$$

Then by choice of $\theta^{*}$ we have $\delta(\Gamma)=\delta_{\theta^{*}}(\Gamma)>s_{\varepsilon}$. Fix $\alpha:=\frac{1}{2}-\frac{s_{\varepsilon}}{2 \delta(\Gamma)}$ and let $r_{0}>0$ such that for all $r \geq r_{0}$ and $t>1$ we have $h(r t) \leq t^{\alpha} h(r)$. In particular, if $b_{\gamma}>\ln \left(r_{0}\right)$, then

$$
h\left(\mathrm{e}^{b_{\gamma}}\right)=h\left(\frac{\mathrm{e}^{b_{\gamma}}}{r_{0}} \cdot r_{0}\right) \leq\left(\frac{\mathrm{e}^{b_{\gamma}}}{r_{0}}\right)^{\alpha} \cdot h\left(r_{0}\right)=\frac{h\left(r_{0}\right)}{r_{0}^{\alpha}} \cdot \mathrm{e}^{\alpha b_{\gamma}} .
$$

Set $\Gamma_{\varepsilon}:=\left\{\gamma \in \Gamma:\left\|\widehat{H}(o, \gamma o)-\theta^{*}\right\|>\varepsilon, b_{\gamma} \geq \ln \left(r_{0}\right)\right\}$. Then

$$
\begin{aligned}
\sum_{\gamma \in \Gamma_{\varepsilon}} \mathrm{e}^{-b_{\gamma}} h\left(\mathrm{e}^{b_{\gamma}}\right) & \leq \frac{h\left(r_{0}\right)}{r_{0}^{\alpha}} \sum_{\gamma \in \Gamma_{\varepsilon}} \mathrm{e}^{\alpha b_{\gamma}} \mathrm{e}^{-b_{\gamma}} \\
& =\frac{h\left(r_{0}\right)}{r_{0}^{\alpha}} \sum_{\gamma \in \Gamma_{\varepsilon}} \mathrm{e}^{-\delta(\Gamma)(1-\alpha) d(o, \gamma o)} .
\end{aligned}
$$

Since $\delta(\Gamma)(1-\alpha)=\delta(\Gamma)\left(\frac{1}{2}+\frac{s_{\varepsilon}}{2 \delta(\Gamma)}\right)=\frac{1}{2} \delta(\Gamma)+\frac{1}{2} s_{\varepsilon}>s_{\varepsilon}$, we conclude that

$$
\sum_{\gamma \in \Gamma_{\varepsilon}} \mathrm{e}^{-b_{\gamma}} h\left(\mathrm{e}^{b_{\gamma}}\right) \text { converges. }
$$


The claim now follows from the fact that the set $\left\{\gamma \in \Gamma: d(o, \gamma o) \leq \ln \left(r_{0}\right) / \delta(\Gamma)\right\}$ is finite.

We finally provide the missing piece in the proof of Theorem A:

Proposition 5.6 The support of the conformal density $\mu=\left(\mu_{x}\right)_{x \in X}$ is contained in $L_{\Gamma} \cap \partial X_{\theta^{*}}$.

Proof. By construction of the map $\mu: X \rightarrow \mathcal{M}^{+}(\partial X)$ in Proposition [5.4] it suffices to show that $\operatorname{supp}\left(\mu_{o}\right) \subset L_{\Gamma} \cap \partial X_{\theta^{*}}$. We already know by definition of $\mu_{o}$ that

$$
\operatorname{supp}\left(\mu_{o}\right) \subset L_{\Gamma} \subset \partial X,
$$

so it suffices to prove that every point $\tilde{\xi} \in \partial X \backslash \partial X_{\theta^{*}}$ possesses an open neighborhood $U \subset \bar{X}$ such that $\mu_{o}(U)=0$. By construction of the measure $\mu_{o}$ as a weak accumulation point of the set $\left\{\mu_{o}^{s}: s>1\right\} \subset \mathcal{M}^{+}(\bar{X})$ for $s \searrow 1$ with $\mu_{o}^{s}$ defined in (20), this is a consequence of Lemma 5.5 .

\section{The generalized Patterson-Sullivan construction}

According to the statement of Theorem A, the classical conformal density constructed in the previous section gives measure zero to the set of limit points of slope different from $\theta^{*}$. In order to obtain measures on an arbitrary $\Gamma$-invariant subset of the limit set we will use a variation of the classical Patterson-Sullivan construction with more degrees of freedom. The idea is to use a weighted version of the Poincaré series in order to get the main contribution from orbit points with direction close to the desired slope $\theta \in E^{+}$. At this point, properties of the exponent of growth and Proposition 4.1 will turn out to be of central importance.

Recall that $\beta_{\theta}$ denotes the directional distance introduced in Definition 3.4. We observe that for any $b=\left(b_{1}, b_{2}, \ldots, b_{r}\right) \in \mathbb{R}^{r}, \theta \in E$ and $\tau \geq 0$ fixed, the sum

$$
P_{\theta}^{s, b, \tau}(x, y)=\sum_{\gamma \in \Gamma} \mathrm{e}^{-s\left(b_{1} d_{1}\left(x_{1}, \gamma_{1} y_{1}\right)+b_{2} d_{2}\left(x_{2}, \gamma_{2} y_{2}\right)+\cdots+b_{r} d_{r}\left(x_{r}, \gamma_{r} y_{r}\right)+\tau\left(d(x, \gamma y)-\beta_{\theta}(x, \gamma y)\right)\right)}
$$

possesses an exponent of convergence which is independent of $x=\left(x_{1}, x_{2}, \ldots, x_{r}\right)$, $y=\left(y_{1}, y_{2}, \ldots, y_{r}\right) \in X$ by the triangle inequalities for $d, d_{1}, d_{2}, \ldots, d_{r}$ and $\beta_{\theta}$. Notice that for $\tau=0$ this is exactly the sum considered by M. Burger [Bur93] in the case of two factors; here we will need to take $\tau>0$ in order to make the contribution of orbit points with direction far away from $\theta$ negligible.

For any $\theta \in E$ and $\tau \geq 0$, we define a region of convergence

$$
\mathcal{R}_{\theta}^{\tau}:=\left\{b=\left(b_{1}, b_{2}, \ldots, b_{r}\right): P_{\theta}^{s, b, \tau}(o, o) \text { has exponent of convergence } s \leq 1\right\} \subset \mathbb{R}^{r}
$$

and its boundary

$$
\partial \mathcal{R}_{\theta}^{\tau}:=\left\{b=\left(b_{1}, b_{2}, \ldots, b_{r}\right): P_{\theta}^{s, b, \tau}(o, o) \text { has exponent of convergence } s=1\right\} \subset \mathbb{R}^{r} .
$$


We recall the definition of the distance vector from (21). In the sequel we will identify $b=\left(b_{1}, b_{2}, \ldots, b_{r}\right)$ with the column vector $b^{\top}$ so that for $q=\left(q_{1}, q_{2}, \ldots, q_{r}\right)^{\top} \in \mathbb{R}^{r}$ we may write

$$
\langle b, q\rangle=b_{1} q_{1}+b_{2} q_{2}+\cdots+b_{r} q_{r} .
$$

The region of convergence possesses the following two properties:

Lemma 6.1 If $\tau \leq \tau^{\prime}$, then $\mathcal{R}_{\theta}^{\tau} \subset \mathcal{R}_{\theta}^{\tau^{\prime}}$.

Proof. Let $\tau \leq \tau^{\prime}, b \in \mathcal{R}_{\theta}^{\tau}$. Then for any $\gamma \in \Gamma$

$$
\mathrm{e}^{-s\left(\langle b, H(o, \gamma o)\rangle+\tau^{\prime}\left(d(o, \gamma o)-\beta_{\theta}(o, \gamma o)\right)\right)} \leq \mathrm{e}^{-s\left(\langle b, H(o, \gamma o)\rangle+\tau\left(d(o, \gamma o)-\beta_{\theta}(o, \gamma o)\right)\right)}
$$

and therefore $P_{\theta}^{s, b, \tau^{\prime}}(o, o) \leq P_{\theta}^{s, b, \tau}(o, o)$. Hence $P_{\theta}^{s, b, \tau^{\prime}}(o, o)$ converges if $s>1$. In particular, $P_{\theta}^{s, b, \tau^{\prime}}(o, o)$ has exponent of convergence less than or equal to 1 .

Lemma 6.2 For any $\tau \geq 0$, the region $\mathcal{R}_{\theta}^{\tau}$ is convex.

Proof. Let $\tau \geq 0, a, b \in \mathcal{R}_{\theta}^{\tau}$ and $t \in[0,1]$. For $\gamma \in \Gamma$ we abbreviate

$$
(t a+(1-t) b)_{\gamma}:=\langle t a+(1-t) b, H(o, \gamma o)\rangle+\tau\left(d(o, \gamma o)-\beta_{\theta}(o, \gamma o)\right)
$$

Then by Hölder's inequality

$$
\sum_{\gamma \in \Gamma} \mathrm{e}^{-s(t a+(1-t) b)_{\gamma}}=\sum_{\gamma \in \Gamma} \mathrm{e}^{-s t a_{\gamma}} \mathrm{e}^{-s(1-t) b_{\gamma}} \leq\left(\sum_{\gamma \in \Gamma} \mathrm{e}^{-s a_{\gamma}}\right)^{t}\left(\sum_{\gamma \in \Gamma} \mathrm{e}^{-s b_{\gamma}}\right)^{1-t} .
$$

The latter sum converges if $s>1$, hence $t a+(1-t) b \in \mathcal{R}_{\theta}^{\tau}$.

With the help of Proposition 4.1 we can describe the region of convergence more precisely. The following result relates the region of convergence $\mathcal{R}_{\theta}^{\tau}$ to the exponent of growth of slope $\theta$.

Lemma 6.3 Let $\theta=\left(\theta_{1}, \theta_{2}, \ldots, \theta_{r}\right) \in E$ and $\tau \geq 0$. If $b=\left(b_{1}, b_{2}, \ldots, b_{r}\right) \in \mathcal{R}_{\theta}^{\tau}$, then

$$
\langle b, \theta\rangle=\sum_{i=1}^{r} b_{i} \theta_{i} \geq \delta_{\theta}(\Gamma) .
$$

Proof. Assume that $\langle b, \theta\rangle\left\langle\delta_{\theta}(\Gamma)\right.$. Then there exists $s>1$ such that $s\langle b, \theta\rangle\left\langle\delta_{\theta}(\Gamma)\right.$. For $H \in \mathbb{R}_{\geq 0}^{r}$ we set

$$
f(H):=s(\langle b, H\rangle+\tau(\|H\|-\langle H, \theta\rangle)),
$$

so the continuous homogeneous function $f: \mathbb{R}_{\geq 0}^{r} \rightarrow \mathbb{R}$ satisfies

$$
f(\theta)=s\langle b, \theta\rangle<\delta_{\theta}(\Gamma) ;
$$


hence according to Proposition 4.1 (a) applied to $D=E$, the sum

$$
\sum_{\gamma \in \Gamma} \mathrm{e}^{-f(H(o, \gamma o))} \quad \text { diverges. }
$$

Since

$$
\begin{aligned}
f(H(o, \gamma o))=s( & b_{1} d_{1}\left(o_{1}, \gamma_{1} o_{1}\right)+b_{2} d_{2}\left(o_{2}, \gamma_{2} o_{2}\right)+\cdots \\
& \left.+b_{r} d_{r}\left(o_{r}, \gamma_{r} o_{r}\right)+\tau\left(d(o, \gamma o)-\beta_{\theta}(o, \gamma o)\right)\right)
\end{aligned}
$$

we have

$$
\sum_{\gamma \in \Gamma} \mathrm{e}^{-f(H(o, \gamma o))}=P_{\theta}^{s, b, \tau}(o, o)
$$

so we get a contradiction to $\left(b_{1}, b_{2}, \ldots, b_{r}\right) \in \mathcal{R}_{\theta}^{\tau}$.

Using the above properties of the region of convergence and Patterson's Lemma 5.1 we are now going to construct $(b, \theta)$-densities as defined in the introduction. Such densities are a natural generalization of $\Gamma$-invariant conformal densities if one wants to measure an arbitrary $\Gamma$-invariant subset of the geometric limit set.

From here on we fix $\theta=\left(\theta_{1}, \theta_{2}, \ldots, \theta_{r}\right) \in E$ such that $L_{\Gamma} \cap \partial X_{\theta} \neq \emptyset, \tau \geq 0$ and a vector $b=\left(b_{1}, b_{2}, \ldots, b_{r}\right) \in \partial \mathcal{R}_{\theta}^{\tau} \subset \mathbb{R}^{r}$; let $\|b\|:=\sqrt{b_{1}^{2}+b_{2}^{2}+\cdots+b_{r}^{2}}$ denote its Euclidean norm. For $x, z \in X$ and $\gamma \in \Gamma$ abbreviate

$$
\begin{aligned}
b(x, z) & :=\langle b, H(x, z)\rangle+\tau\left(d(x, z)-\beta_{\theta}(x, z)\right), \\
b_{\gamma} & :=b(o, \gamma o) .
\end{aligned}
$$

Then $\beta_{\theta}(x, z) \geq 0$ and the Cauchy-Schwarz inequality

$$
|\langle b, H(x, z)\rangle| \leq\|b\| \cdot\|H(x, z)\|=\|b\| \cdot d(x, z)
$$

give the important rough estimate

$$
d(x, z) \geq \frac{1}{\|b\|+\tau} \cdot b(x, z)
$$

notice that since the $b_{i}$ may be negative numbers, a converse inequality does not hold in general. In other words, there may exist sequences $\left(z_{n}\right) \subset X$ such that $b\left(o, z_{n}\right)$ remains bounded even if $d\left(o, z_{n}\right)$ tends to infinity. The following lemma gives a condition which ensures that $b\left(o, z_{n}\right)$ tends to infinity if $d\left(o, z_{n}\right)$ does.

Lemma 6.4 If $\delta_{\theta}(\Gamma)>0$ and $\left(z_{n}\right) \subset X$ is a sequence converging to a point in $\partial X_{\theta}$, then

$$
b\left(o, z_{n}\right) \rightarrow \infty \text { as } n \rightarrow \infty .
$$

Proof. Lemma 6.3 states that for $b=\left(b_{1}, b_{2}, \ldots, b_{r}\right) \in \partial \mathcal{R}_{\theta}^{\tau}$ the inequality

$$
\langle b, \theta\rangle \geq \delta_{\theta}(\Gamma)
$$

holds; since $\delta_{\theta}(\Gamma)>0$ and the map $\hat{\theta} \mapsto\langle b, \hat{\theta}\rangle$ is continuous, there exists $\varepsilon>0$ such that for all $\hat{\theta} \in E$ with $\|\hat{\theta}-\theta\|<\varepsilon$ we have

$$
\langle b, \hat{\theta}\rangle \geq q>0 .
$$


Since $\left(z_{n}\right) \subset X$ converges to a point in $\partial X_{\theta}$, for all $n$ sufficiently large we have

$$
\left\|\theta-\widehat{H}\left(o, z_{n}\right)\right\|<\varepsilon, \quad \text { hence }\left\langle b, H\left(o, z_{n}\right)\right\rangle \geq q \cdot d\left(o, z_{n}\right) .
$$

Summarizing, we get for all $n$ sufficiently large

$$
b\left(o, z_{n}\right)=\left\langle b, H\left(o, z_{n}\right)\right\rangle+\tau \underbrace{\left(d\left(o, z_{n}\right)-\beta_{\theta}\left(o, z_{n}\right)\right)}_{\geq 0} \geq q \cdot d\left(o, z_{n}\right) .
$$

Let $h$ be a function as in Patterson's Lemma 5.1 and recall the definition of the distance vector from (2). As in Section 5 we will construct a family of orbital measures on $\bar{X}$ in the following way: If $D$ denotes the unit Dirac point measure, then for $x \in X$ and $s>1$ we put

$$
\mu_{x}^{s}:=\frac{1}{P^{s}} \sum_{\gamma \in \Gamma} \mathrm{e}^{-s b(x, \gamma o)} h\left(\mathrm{e}^{b(x, \gamma o)}\right) D(\gamma o), \quad \text { where } \quad P^{s}=\sum_{\gamma \in \Gamma} \mathrm{e}^{-s b_{\gamma}} h\left(\mathrm{e}^{b_{\gamma}}\right) .
$$

As in the classical case, these measures are $\Gamma$-equivariant by construction, but now they depend on the additional parameters $\theta \in E, \tau \geq 0$ and $b=\left(b_{1}, b_{2}, \ldots, b_{r}\right) \in \partial \mathcal{R}_{\theta}^{\tau}$. For $x, y \in X$ and $s>1$ the measures $\mu_{x}^{s}$ and $\mu_{y}^{s}$ are absolutely continuous with respect to each other with Radon Nikodym derivative

$$
\frac{\mathrm{d} \mu_{x}^{s}}{\mathrm{~d} \mu_{y}^{s}}(z)=\frac{\mathrm{e}^{-s b(x, z)} h\left(\mathrm{e}^{b(x, z)}\right)}{\mathrm{e}^{-s b(y, z)} h\left(\mathrm{e}^{b(y, z)}\right)}, \quad z \in \operatorname{supp}\left(\mu_{y}^{s}\right)
$$

which again is the function $g_{x, y}^{s}$ defined by (22) in Lemma 5.2 , but now with the continuous map $b: X \times X \rightarrow \mathbb{R}$ given by (24). For $x=\left(x_{1}, x_{2}, \ldots, x_{r}\right), y=\left(y_{1}, y_{2}, \ldots, y_{r}\right)$, $z=\left(z_{1}, z_{2}, \ldots, z_{r}\right) \in X$ we further have the estimate

$$
\begin{aligned}
|b(x, z)-b(y, z)|= & \mid\langle b, H(x, z)-H(y, z)\rangle \\
& +\tau(d(x, z)-d(y, z))-\tau\left(\beta_{\theta}(x, z)-\beta_{\theta}(y, z)\right) \mid \\
\leq & \|b\| \cdot\|H(x, z)-H(y, z)\|+2 \tau d(x, y) \leq(\|b\|+2 \tau) d(x, y),
\end{aligned}
$$

which is (21) with constant $C=\|b\|+2 \tau$. So the conclusion of Lemma 5.2 remains true for our new function $g_{x, y}^{s}: X \rightarrow \mathbb{R}$. Unfortunately an analogous statement of Lemma 5.3 does not hold, because in general $g_{x, y}^{s}$ cannot be extended continuously to the whole geometric boundary. However, the following statement will be sufficient for our purposes:

Lemma 6.5 If $\theta \in E^{+}$and $\delta_{\theta}(\Gamma)>0$, then for fixed $x, y \in X$ and $s>0$ the function

$$
g_{x, y}^{s}: X \rightarrow \mathbb{R}, \quad z \mapsto \frac{\mathrm{e}^{-s b(x, z)} h\left(\mathrm{e}^{b(x, z)}\right)}{\mathrm{e}^{-s b(y, z)} h\left(\mathrm{e}^{b(y, z)}\right)}
$$

extends continuously to $X \cup \partial X_{\theta}$. Moreover, if $x=\left(x_{1}, x_{2}, \ldots, x_{r}\right), y=\left(y_{1}, y_{2}, \ldots, y_{r}\right)$ and $\left(z_{n}\right) \subset X$ is a sequence converging to $\tilde{\eta}=\left(\eta_{1}, \eta_{2}, \ldots, \eta_{r}, \theta\right) \in \partial X_{\theta} \subset \partial X^{r e g}$, then

$$
\lim _{n \rightarrow \infty} g_{x, y}^{s}\left(z_{n}\right)=\mathrm{e}^{-s\left(b_{1} \beta_{\eta_{1}}\left(x_{1}, y_{1}\right)+b_{2} \beta_{\eta_{2}}\left(x_{2}, y_{2}\right)+\cdots+b_{r} \beta_{\eta_{r}}\left(x_{r}, y_{r}\right)\right)} .
$$


Proof. We first notice that if $\left(z_{n}\right)=\left(z_{n, 1}, z_{n, 2}, \ldots, z_{n, r}\right) \subset X$ is a sequence converging to a point $\tilde{\eta}=\left(\eta_{1}, \eta_{2}, \ldots, \eta_{r}, \hat{\theta}\right) \in \partial X_{\hat{\theta}} \subset \partial X^{r e g}$ with $\hat{\theta}=\left(\hat{\theta}_{1}, \hat{\theta}_{2}, \ldots, \hat{\theta}_{r}\right) \in E^{+}$, then for all $i \in\{1,2, \ldots, r\}$

$$
\begin{array}{rll}
d_{i}\left(x_{i}, z_{n, i}\right)-d_{i}\left(\cdot, z_{n, i}\right) & \text { converges to } & \beta_{\eta_{i}}\left(x_{i}, \cdot\right), \\
\text { and } d\left(x, z_{n}\right)-d\left(\cdot, z_{n}\right) & \text { converges to } & \beta_{\tilde{\eta}}(x, \cdot)
\end{array}
$$

uniformly on compact sets in $X_{i}$ respectively $X=X_{1} \times X_{2} \times \cdots \times X_{r}$. Together with Definition 3.4 of the directional distance this implies in particular that

$$
\beta_{\theta}\left(x, z_{n}\right)-\beta_{\theta}\left(\cdot, z_{n}\right) \quad \text { converges to } \sum_{i=1}^{r} \hat{\theta}_{i} \cdot \beta_{\eta_{i}}\left(x_{i}, \cdot\right) .
$$

Moreover, if $\theta_{i} \in \mathbb{R}_{>0}, i \in\{1,2, \ldots, r\}$, denotes the $i$-th coordinate of $\theta \in E^{+}$, then Lemma 3.3 gives

$$
\beta_{\widetilde{\eta}}(x, y)=\sum_{i=1}^{r} \theta_{i} \cdot \beta_{\eta_{i}}\left(x_{i}, y_{i}\right)
$$

So we conclude

$$
\begin{aligned}
\lim _{n \rightarrow \infty}\left(b\left(x, z_{n}\right)-b\left(y, z_{n}\right)\right) & =\sum_{i=1}^{r} b_{i} \beta_{\eta_{i}}\left(x_{i}, y_{i}\right)+\tau \beta_{\widetilde{\eta}}(x, y)-\tau\left(\sum_{i=1}^{r} \hat{\theta}_{i} \cdot \beta_{\eta_{i}}\left(x_{i}, y_{i}\right)\right) \\
& =\sum_{i=1}^{r} b_{i} \beta_{\eta_{i}}\left(x_{i}, y_{i}\right)+\tau\left(\sum_{i=1}^{r}\left(\theta_{i}-\hat{\theta}_{i}\right) \cdot \beta_{\eta_{i}}\left(x_{i}, y_{i}\right)\right)
\end{aligned}
$$

and therefore in the case $\hat{\theta}=\theta$

$$
\begin{aligned}
\lim _{n \rightarrow \infty} \frac{\mathrm{e}^{-s b\left(x, z_{n}\right)}}{\mathrm{e}^{-s b\left(y, z_{n}\right)}} & =\lim _{n \rightarrow \infty} \mathrm{e}^{-s\left(b\left(x, z_{n}\right)-b\left(y, z_{n}\right)\right)} \\
& =\mathrm{e}^{-s\left(b_{1} \beta_{\eta_{1}}\left(x_{1}, y_{1}\right)+b_{2} \beta_{\eta_{2}}\left(x_{2}, z_{2}\right)+\cdots+b_{r} \beta_{\eta_{r}}\left(x_{r}, z_{r}\right)\right)} .
\end{aligned}
$$

As in the proof of Lemma 5.3 we next show that for any sequence $\left(z_{n}\right) \subset X$ with $b\left(o, z_{n}\right) \rightarrow \infty$ we have

$$
\lim _{n \rightarrow \infty} \frac{h\left(\mathrm{e}^{b\left(x, z_{n}\right)}\right)}{h\left(\mathrm{e}^{b\left(y, z_{n}\right)}\right)}=1
$$

Let $\varepsilon>0$ be arbitrarily small and fix $\alpha<\frac{\ln (1+\varepsilon)}{(\|b\|+2 \tau) d(x, y)}$. Then by Patterson's Lemma 5.1 (ii) there exists $r_{0}>0$ such that for $r \geq r_{0}$ and $t>1$

$$
\frac{h(r t)}{h(r)} \leq t^{\alpha}
$$

So for all $z \in X$ with $b(x, z) \geq \ln \left(r_{0}\right)$ and $b(y, z) \geq \ln \left(r_{0}\right)$ we get

$$
\mathrm{e}^{-\alpha|b(x, z)-b(y, z)|} \leq \frac{h\left(\mathrm{e}^{b(x, z)}\right)}{h\left(\mathrm{e}^{b(y, z)}\right)} \leq \mathrm{e}^{\alpha|b(x, z)-b(y, z)|} .
$$


From the estimate (27) and by choice of $\alpha$ we have

$$
\mathrm{e}^{-\alpha(\|b\|+2 \tau) d(x, y)} \leq \frac{h\left(\mathrm{e}^{b(x, z)}\right)}{h\left(\mathrm{e}^{b(y, z)}\right)} \leq \mathrm{e}^{\alpha(\|b\|+2 \tau) d(x, y)}<1+\varepsilon .
$$

Using again the inequality $\mathrm{e}^{t}+\mathrm{e}^{-t} \geq 2$ we get as a lower bound

$$
\frac{h\left(\mathrm{e}^{b(x, z)}\right)}{h\left(\mathrm{e}^{b(y, z)}\right)} \geq \mathrm{e}^{-\alpha(\|b\|+2 \tau) d(x, y)} \geq 2-\mathrm{e}^{\alpha(\|b\|+2 \tau) d(x, y)}>2-(1+\varepsilon)=1-\varepsilon .
$$

So for $n$ sufficiently large

$$
\left|1-\frac{h\left(\mathrm{e}^{b\left(x, z_{n}\right)}\right)}{h\left(\mathrm{e}^{b\left(y, z_{n}\right)}\right)}\right|<\varepsilon ;
$$

the problem here is that $b\left(o, z_{n}\right)$ may remain bounded even if $d\left(o, z_{n}\right)$ tends to infinity. However, Lemma 6.4 ensures that this does not happen for sequences $\left(z_{n}\right) \subset X$ converging to a point in $\partial X_{\theta}$.

We emphasize again that unlike in the case $b(x, z)=\delta(\Gamma) d(x, z)$ for the classical construction, the continuous function $g_{x, y}^{s}$ considered here need not extend continuously to the whole geometric boundary. One obstruction is the fact that if a sequence $\left(z_{n}\right)=\left(z_{n, 1}, z_{n, 2}, \ldots, z_{n, r}\right)$ converges to a point in $\partial X^{\text {sing }}$, then its projections to one or more factors $X_{i}$ need not converge. And even if a sequence $\left(z_{n}\right)$ converges to a regular boundary point with a slope different from $\theta, b\left(o, z_{n}\right)$ may remain bounded and hence the quotient $\frac{h\left(\mathrm{e}^{b\left(x, z_{n}\right)}\right)}{h\left(\mathrm{e}^{b\left(y, z_{n}\right)}\right)}$ does not necessarily tend to one.

So in general - for arbitrary $b=\left(b_{1}, b_{2}, \ldots, b_{r}\right) \in \partial \mathcal{R}_{\theta}^{\tau}$ - there is no analogon of Proposition 5.4. However, we still have some freedom in choosing appropriate parameters $b=\left(b_{1}, b_{2}, \ldots, b_{r}\right) \in \mathbb{R}^{r}$, which can be done as follows: Since the homogeneous extension $\Psi_{\Gamma}: \mathbb{R}_{\geq 0}^{r} \rightarrow \mathbb{R}$ of the exponent of growth is concave and upper semi-continuous, it is continuous on the closed convex cone

$$
\ell_{\Gamma}:=\left\{H \in \mathbb{R}_{\geq 0}^{r}: \Psi_{\Gamma}(H) \geq 0\right\}
$$

So for any $p \in \mathbb{R}_{\geq 0}^{r}$ in the relative interior of the intersection of $\ell_{\Gamma}$ with the vector subspace of $\mathbb{R}^{r}$ it spans - which thanks to Theorem 7.9 in Lin13 is equal to the set of points $p \in \mathbb{R}_{\geq 0}^{r}$ with $\Psi_{\Gamma}(p)>0$ - there exists a linear functional $\Phi$ on $\mathbb{R}^{r}$ such that

$$
\Phi(p)=\Psi_{\Gamma}(p), \quad \text { and } \quad \Phi(q) \geq \Psi_{\Gamma}(q) \quad \text { for all } \quad q \in \mathbb{R}_{\geq 0}^{r} ;
$$

if $\Psi_{\Gamma}$ is differentiable at $p$, then this linear functional $\Phi$ is unique, but in general it is not. For obvious reasons we will call a linear functional $\Phi$ satisfying (28) tangent to $\Psi_{\Gamma}$ at the point $p \in \mathbb{R}_{\geq 0}^{r}$. Similarly, we will call a vector $b=\left(b_{1}, b_{2}, \ldots, b_{r}\right) \in \mathbb{R}^{r}$ tangent to $\Psi_{\Gamma}$ at $p \in \mathbb{R}_{\geq 0}^{r}$, if the linear functional

$$
\Phi: \mathbb{R}^{r} \rightarrow \mathbb{R}, \quad q \mapsto\langle b, q\rangle
$$

is tangent to $\Psi_{\Gamma}$ at the point $p$. Notice that if $b$ is tangent to $\Psi_{\Gamma}$ at a point $\theta \in E$, then Proposition 4.1 implies $b \in \partial \mathcal{R}_{\theta}^{\tau}$. 
It will turn out in the sequel that the choice of $b=\left(b_{1}, b_{2}, \ldots, b_{r}\right) \in \mathbb{R}^{r}$ tangent to $\Psi_{\Gamma}$ at $\theta$ is the suitable one. The following key proposition analogous to Lemma 5.5 implies that with this choice any weak accumulation point of the set of measures $\left\{\mu_{o}^{s}: s>1\right\} \subset \mathcal{M}^{+}(\bar{X})$ as $s \searrow 1$ is supported in $\partial X_{\theta}$. It is therefore the key ingredient in the construction of orbital measures with support in a single $\Gamma$-invariant subset $L_{\Gamma} \cap \partial X_{\theta} \subset \partial X$.

Proposition 6.6 Fix $\theta \in E^{+}$such that $\delta_{\theta}(\Gamma)>0$, and let $b=\left(b_{1}, b_{2}, \ldots, b_{r}\right) \in \mathbb{R}^{r}$ be a vector tangent to $\Psi_{\Gamma}$ at $\theta$. Then for all $\tau>0$ and for all $\varepsilon>0$

$$
\sum_{\substack{\gamma \in \Gamma \\(o, \gamma o)-\theta \|>\varepsilon}} \mathrm{e}^{-b_{\gamma}} h\left(\mathrm{e}^{b_{\gamma}}\right)<\infty,
$$

where $b_{\gamma}$ is defined in (24) and $h$ is a function as in Patterson's Lemma 5.1.

Proof. Since $b=\left(b_{1}, b_{2}, \ldots, b_{r}\right) \in \mathbb{R}^{r}$ is tangent to $\Psi_{\Gamma}$ at $\theta$ and $\Psi_{\Gamma}(\theta)=\delta_{\theta}(\Gamma)$ we have

$$
\langle b, \theta\rangle=\delta_{\theta}(\Gamma) \quad \text { and } \quad\langle b, \hat{\theta}\rangle \geq \delta_{\hat{\theta}}(\Gamma) \quad \text { for all } \hat{\theta} \in E .
$$

We fix $\tau>0$ and let $\varepsilon>0$ be arbitrary. Since the sum is non-increasing when $\varepsilon$ gets bigger, we may further assume that $\varepsilon<1$.

We set $D:=\{\hat{\theta} \in E:\|\hat{\theta}-\theta\|>\varepsilon\}, s_{\varepsilon}:=\max \{|\langle b, \hat{\theta}\rangle|: \hat{\theta} \in \bar{D}\} \geq 0$, and fix

$$
\alpha<\frac{\tau \varepsilon^{2}}{\tau \varepsilon^{2}+2 s_{\varepsilon}} \leq 1
$$

By Patterson's Lemma 5.1 (ii) there exists $r_{0}>0$ such that for all $t>1$ we have $h\left(r_{0} t\right) \leq t^{\alpha} h\left(r_{0}\right)$. So $b_{\gamma}>\ln \left(r_{0}\right)$ implies

$$
h\left(\mathrm{e}^{b_{\gamma}}\right)=h\left(\frac{\mathrm{e}^{b_{\gamma}}}{r_{0}} \cdot r_{0}\right) \leq\left(\frac{\mathrm{e}^{b_{\gamma}}}{r_{0}}\right)^{\alpha} h\left(r_{0}\right) .
$$

We therefore have

$$
\sum_{\substack{\gamma \in \Gamma \\ \widehat{H}(o, \gamma o)-\theta \|>\varepsilon \\ b_{\gamma}>\ln \left(r_{0}\right)}} \mathrm{e}^{-b_{\gamma}} h\left(\mathrm{e}^{b_{\gamma}}\right) \leq \frac{h\left(r_{0}\right)}{r_{0}^{\alpha}} \sum_{\substack{\gamma \in \Gamma \\\|\hat{H}(o, \gamma o)-\theta\|>\varepsilon \\ b_{\gamma}>\ln \left(r_{0}\right)}} \mathrm{e}^{-(1-\alpha) b_{\gamma}}<\infty,
$$

by Proposition 4.1. Indeed, the continuous homogeneous function $f: \mathbb{R}_{\geq 0}^{r} \rightarrow \mathbb{R}$ defined by

$$
f(H):=(1-\alpha)(\langle b, H\rangle+\tau(\|H\|-\langle H, \theta\rangle))
$$

satisfies $f(H(o, \gamma o))=(1-\alpha) b_{\gamma}$ for all $\gamma \in \Gamma$, and

$$
f(\hat{\theta})>\delta_{\hat{\theta}}(\Gamma) \text { for all } \hat{\theta} \in \bar{D}=\{\hat{\theta} \in E:\|\hat{\theta}-\theta\| \geq \varepsilon\}
$$


by the following estimate:

$$
\begin{aligned}
f(\hat{\theta}) & =(1-\alpha)(\langle b, \hat{\theta}\rangle+\tau(1-\langle\hat{\theta}, \theta\rangle))=(1-\alpha)\langle b, \hat{\theta}\rangle+(1-\alpha) \frac{\tau}{2} \underbrace{\|\hat{\theta}-\theta\|^{2}}_{\geq \varepsilon^{2}} \\
& \geq\langle b, \hat{\theta}\rangle-\alpha|\langle b, \hat{\theta}\rangle|+(1-\alpha) \tau \frac{\varepsilon^{2}}{2} \geq\langle b, \hat{\theta}\rangle+\tau \frac{\varepsilon^{2}}{2}-\alpha\left(|\langle b, \hat{\theta}\rangle|+\tau \frac{\varepsilon^{2}}{2}\right) \\
& >\langle b, \hat{\theta}\rangle+\tau \frac{\varepsilon^{2}}{2}-\frac{\tau \varepsilon^{2}}{\tau \varepsilon^{2}+2 s_{\varepsilon}}(\underbrace{|\langle b, \hat{\theta}\rangle|}_{\leq s_{\varepsilon}}+\tau \frac{\varepsilon^{2}}{2}) \geq\langle b, \hat{\theta}\rangle \geq \delta_{\hat{\theta}}(\Gamma) .
\end{aligned}
$$

So it remains to show that the sum

$$
\sum_{\substack{\gamma \in \Gamma \\ \hat{H}(o, \gamma o)-\theta \|>\varepsilon \\ b_{\gamma} \leq \ln \left(r_{0}\right)}} \mathrm{e}^{-b_{\gamma}} h\left(\mathrm{e}^{b_{\gamma}}\right)
$$

is finite. This is not as trivial as in Lemma 5.5. because due to the fact that the coordinates $b_{i}, i \in\{1,2, \ldots, r\}$, need not be positive, $b_{\gamma_{n}}$ might remain bounded even if $d\left(o, \gamma_{n} o\right)$ tends to infinity. However, we can argue in the following way: By Property (a) of the exponent of growth the set of slopes of limit points

$$
P_{\Gamma}:=\left\{\theta^{\prime} \in E: L_{\Gamma} \cap \partial X_{\theta^{\prime}} \neq \emptyset\right\}
$$

satisfies

$$
P_{\Gamma}=\left\{\theta^{\prime} \in E: \delta_{\theta}(\Gamma) \geq 0\right\} .
$$

Since the map $E \rightarrow \mathbb{R}, \hat{\theta} \mapsto\langle b, \hat{\theta}\rangle$ is continuous and satisfies $\langle b, \hat{\theta}\rangle \geq \delta_{\hat{\theta}}(\Gamma)$ for all $\hat{\theta} \in E$, there exists $\alpha>0$ such that for all $\gamma \in \Gamma$ with $\widehat{H}(o, \gamma o)$ in the $\alpha$-neighborhood of $P_{\Gamma}$ defined by

$$
\left\{\hat{\theta} \in E:\left\|\hat{\theta}-\theta^{\prime}\right\|<\alpha \quad \text { for some } \quad \theta^{\prime} \in P_{\Gamma}\right\}
$$

we have

$$
\langle b, \widehat{H}(o, \gamma o)\rangle>-\frac{\tau \varepsilon^{2}}{4} .
$$

Hence for all $\gamma \in \Gamma$ with $\|\widehat{H}(o, \gamma o)-\theta\|>\varepsilon$ and $\left\|\widehat{H}(o, \gamma o)-\theta^{\prime}\right\|<\alpha$ for some $\theta^{\prime} \in P_{\Gamma}$ we get

$$
\begin{aligned}
b_{\gamma} & =d(o, \gamma o)(\langle b, \widehat{H}(o, \gamma o)\rangle+\tau \underbrace{(1-\langle\widehat{H}(o, \gamma o), \theta\rangle)}_{\geq \varepsilon^{2} / 2}) \\
& >d(o, \gamma o)\left(-\frac{\tau \varepsilon^{2}}{4}+\tau \frac{\varepsilon^{2}}{2}\right)=\frac{\tau \varepsilon^{2}}{4} d(o, \gamma o) .
\end{aligned}
$$

So $b_{\gamma} \leq \ln \left(r_{0}\right)$ implies $d(o, \gamma o) \leq \frac{4}{\tau \varepsilon^{2}} \ln \left(r_{0}\right)$, hence the set

$$
\left\{\gamma \in \Gamma:\|\widehat{H}(o, \gamma o)-\theta\|>\varepsilon,\left\|\widehat{H}(o, \gamma o)-\theta^{\prime}\right\|<\alpha \text { for some } \theta^{\prime} \in P_{\Gamma}, b_{\gamma} \leq \ln \left(r_{0}\right)\right\}
$$

is finite. 
Now assume the remaining set

$$
\Gamma^{\prime}:=\left\{\gamma \in \Gamma:\|\widehat{H}(o, \gamma o)-\theta\|>\varepsilon,\left\|\widehat{H}(o, \gamma o)-\theta^{\prime}\right\| \geq \alpha \text { for all } \theta^{\prime} \in P_{\Gamma}, b_{\gamma} \leq \ln \left(r_{0}\right)\right\}
$$

is infinite. Then there exists an accumulation point of the orbit $\Gamma^{\prime} o$ which cannot belong to the geometric boundary $\partial X$ of $X$ by definition of the set $P_{\Gamma}$. Since $\Gamma^{\prime}$ is discrete, the accumulation point cannot belong to $X$ either, so the only possibility is that $\Gamma^{\prime}$ is finite.

Notice that the proof above also works in the case $\theta=\left(\theta_{1}, \theta_{2}, \ldots, \theta_{r}\right) \in E \backslash E^{+}$. However, in general the vector $b=\left(b_{1}, b_{2}, \ldots, b_{r}\right)$ tangent to $\Psi_{\Gamma}$ at $\theta$ does not satisfy $b_{i}=0$ for all $i \in\{1,2, \ldots, r\}$ with $\theta_{i}=0$, which would be necessary for a welldefined Radon Nikodym derivative of the $(b, \theta)$-density. Hence for the construction of $(b, \theta)$-densities we have to restrict ourselves to $\theta \in E^{+}$.

From here on we therefore fix a slope $\theta=\left(\theta_{1}, \theta_{2}, \ldots, \theta_{r}\right) \in E^{+}$such that $\Psi_{\Gamma}(\theta)=\delta_{\theta}(\Gamma)>0$; hence in particular we have $L_{\Gamma} \cap \partial X_{\theta} \neq \emptyset$. Using the previous proposition we are finally able to construct a $(b, \theta)$-density according to Definition 1.2 ,

To that end we further choose $b=\left(b_{1}, b_{2}, \ldots, b_{r}\right) \in \mathbb{R}^{r}$ tangent to $\Psi_{\Gamma}$ at $\theta$ and fix $\tau>0$. By Proposition 6.6 and arguments analogous to the proof of Proposition 5.6 any weak accumulation point $\mu_{o}$ of $\mu_{o}^{s}$ (defined with these parameters) as $s \searrow 1$ satisfies $\operatorname{supp}\left(\mu_{o}\right) \subset L_{\Gamma} \cap \partial X_{\theta}$.

The last step in the proof of Theorem B from the introduction needs an analogon of Proposition 5.4, we have to show that a suitable choice of a weak accumulation point for each of the sets

$$
\left\{\mu_{x}^{s}: s \in(1,2]\right\} \subset \mathcal{M}^{+}(\bar{X}), \quad x \in X,
$$

produces a $(b, \theta)$-density. We recall that for $\tilde{\eta} \in \partial X$ the Busemann vector $\mathcal{B}_{\tilde{\eta}}$ was defined in (111).

Proposition 6.7 Let $\left(s_{j}\right) \subset \mathbb{R}, s_{j} \searrow 1$ be a sequence such that $\mu_{o}^{s_{j}}$ converges weakly to $\mu_{o}$, and $x \in X$ arbitrary. Then the sequence of measures $\mu_{x}^{s_{j}}$ converges weakly to a measure $\mu_{x} \in \mathcal{M}^{+}(\partial X)$ with $\operatorname{supp}\left(\mu_{x}\right) \subset L_{\Gamma} \cap \partial X_{\theta}$ and

$$
\frac{d \mu_{x}}{d \mu_{o}}(\tilde{\eta})=\mathrm{e}^{\left\langle b, \mathcal{B}_{\tilde{\eta}}(o, x)\right\rangle} \quad \text { for all } \tilde{\eta} \in \operatorname{supp}\left(\mu_{o}\right) .
$$

Proof. Let $f \in \mathrm{C}^{0}(\bar{X})$ with $\|f\|_{\infty}<\infty$ be arbitrary, $s>1$ and denote $g_{x, o}^{s}: X \rightarrow \mathbb{R}$ the continuous function defined by (22) in Lemma 5.2, which extends continuously to $X \cup \partial X_{\theta}$ by Lemma 6.5. By (26) we have for all $s>1$ and for all $z \in \operatorname{supp}\left(\mu_{o}^{s}\right)$

$$
\frac{\mathrm{d} \mu_{x}^{s}}{\mathrm{~d} \mu_{y}^{s}}(z)=\frac{\mathrm{e}^{-s b(x, z)} h\left(\mathrm{e}^{b(x, z)}\right)}{\mathrm{e}^{-s b(y, z)} h\left(\mathrm{e}^{b(y, z)}\right)}=g_{x, o}^{s}(z),
$$

hence

$$
\int_{X \cup \partial X_{\theta}} f(z) \mathrm{d} \mu_{x}^{s}(z)=\int_{X \cup \partial X_{\theta}} f(z) g_{x, o}^{s}(z) \mathrm{d} \mu_{o}^{s}(z) .
$$


We claim that for any sequence $\left(s_{j}\right) \searrow 1$ such that $\mu_{o}^{s_{j}}$ converges weakly to $\mu_{o}$ we have

$$
\lim _{j \rightarrow \infty} \int_{\bar{X}} f(z) \mathrm{d} \mu_{x}^{s_{j}}(z)=\int_{\bar{X}} f(\tilde{\eta}) \mathrm{e}^{\left\langle b, \mathcal{B}_{\tilde{\eta}}(o, x)\right\rangle} \mathrm{d} \mu_{o}(\tilde{\eta}) ;
$$

so the measure $\mu_{x}$ defined by

$$
\frac{\mathrm{d} \mu_{x}}{\mathrm{~d} \mu_{o}}(\tilde{\eta})=\mathrm{e}^{\left\langle b, \mathcal{B}_{\tilde{\eta}}(o, x)\right\rangle} \quad \text { for all } \tilde{\eta} \in \operatorname{supp}\left(\mu_{o}\right)
$$

is the weak limit of the sequence of measures $\mu_{x}^{s_{j}}$. Then in particular we have

$$
\operatorname{supp}\left(\mu_{x}\right) \subset \operatorname{supp}\left(\mu_{o}\right) \subset L_{\Gamma} \cap \partial X_{\theta} .
$$

In order to prove $(*)$ we notice that by Lemma 6.5 we have for $\tilde{\eta} \in \operatorname{supp}\left(\mu_{o}\right) \subset \partial X_{\theta}$

$$
\mathrm{e}^{\left\langle b, \mathcal{B}_{\tilde{\eta}}(o, x)\right\rangle}=g_{x, o}^{1}(\tilde{\eta}) ;
$$

moreover, using the fact that the support of any measure in the weak closure of the set $\left\{\mu_{x}^{s}: s \in(1,2]\right\} \subset \mathcal{M}^{+}(\bar{X})$ is contained in $X \cup \partial X_{\theta}$, we estimate

$$
\begin{aligned}
\mid \int_{\bar{X}} f(z) \mathrm{d} \mu_{x}^{s_{j}}(z) & -\int_{\bar{X}} f(\tilde{\eta}) \mathrm{e}^{\left\langle b, \mathcal{B}_{\tilde{\eta}}(o, x)\right\rangle} \mathrm{d} \mu_{o}(\tilde{\eta}) \mid \\
& =\left|\int_{X \cup \partial X_{\theta}} f(z) \mathrm{d} \mu_{x}^{s_{j}}(z)-\int_{\partial X_{\theta}} f(\tilde{\eta}) \mathrm{e}^{\left\langle b, \mathcal{B}_{\tilde{\eta}}(o, x)\right\rangle} \mathrm{d} \mu_{o}(\tilde{\eta})\right| \\
& =\left|\int_{X \cup \partial X_{\theta}} f(z) g_{x, o}^{s_{j}}(z) \mathrm{d} \mu_{o}^{s_{j}}(z)-\int_{\partial X_{\theta}} f(\tilde{\eta}) g_{x, o}^{1}(\tilde{\eta}) \mathrm{d} \mu_{o}(\tilde{\eta})\right| \\
& \leq\left|\int_{X \cup \partial X_{\theta}} f(z) g_{x, o}^{s_{j}}(z) \mathrm{d} \mu_{o}^{s_{j}}(z)-\int_{X \cup \partial X_{\theta}} f(z) g_{x, o}^{1}(z) \mathrm{d} \mu_{o}^{s_{j}}(z)\right| \\
& +\left|\int_{X \cup \partial X_{\theta}} f(z) g_{x, o}^{1}(z) \mathrm{d} \mu_{o}^{s_{j}}(z)-\int_{X \cup \partial X_{\theta}} f(\tilde{\eta}) g_{x, o}^{1}(\tilde{\eta}) \mathrm{d} \mu_{o}(\tilde{\eta})\right| .
\end{aligned}
$$

Since $f \cdot g_{x, o}^{1}$ is a bounded and continuous function on $X \cup \partial X_{\theta}$, and $\mu_{o}^{s_{j}}$ converges weakly to $\mu_{o}$, the second term tends to zero as $j$ tends to infinity.

For the first term we argue as in the proof of Proposition 5.4. By the estimate (27) the assumption (21) on $b$ in Lemma 5.2 is satisfied with $C=\|b\|+2 \tau$, hence by definition of the measure $\mu_{o}^{s_{j}}$ and by Lemma 5.2 we have

$$
\begin{aligned}
\mid \int_{X \cup \partial X_{\theta}} f(z) g_{x, o}^{s_{j}}(z) \mathrm{d} \mu_{o}^{s_{j}}(z) & -\int_{X \cup \partial X_{\theta}} f(z) g_{x, o}^{1}(z) \mathrm{d} \mu_{o}^{s_{j}}(z) \mid \\
& =\left|\int_{X \cup \partial X_{\theta}} f(z)\left(g_{x, o}^{s_{j}}(z)-g_{x, o}^{1}(z)\right) \mathrm{d} \mu_{o}^{s_{j}}(z)\right| \\
& =\frac{1}{P^{s_{j}}} \sum_{\gamma \in \Gamma} f(\gamma o)\left(g_{x, o}^{s_{j}}(\gamma o)-g_{x, o}^{1}(\gamma o)\right) \mathrm{e}^{-s_{j} b(o, \gamma o)} h\left(\mathrm{e}^{b(o, \gamma o)}\right) \\
& \leq\|f\|_{\infty}\left\|g_{x, o}^{s_{j}}-g_{x, o}^{1}\right\|_{\infty} \rightarrow 0 \quad \text { as } \quad j \rightarrow \infty .
\end{aligned}
$$


So given a slope $\theta \in E^{+}$satisfying $\Psi_{\Gamma}(\theta)=\delta_{\theta}(\Gamma)>0$ our construction with $b=\left(b_{1}, b_{2}, \ldots, b_{r}\right) \in \mathbb{R}^{r}$ tangent to $\Psi_{\Gamma}$ at the point $\theta$ and $\tau>0$ arbitrary produces the desired $(b, \theta)$-density. This proves Theorem B from the introduction.

\section{Properties of $(b, \theta)$-densities}

In this section we will study properties of $(b, \theta)$-densities using the shadow lemma Theorem [7.2, If not otherwise specified we allow $\theta \in E$.

LeMmA 7.1 Let $\mu$ be $a(b, \theta)$-density, and $x \in X$. If $\widetilde{U} \subset \partial X$ is an open neighborhood of a limit point $\tilde{\xi} \in \partial X_{\theta}$, then $\mu_{x}(\widetilde{U})>0$.

Proof. Let $\widetilde{U} \subset \partial X$ be an open neighborhood of a limit point $\tilde{\xi} \in \partial X_{\theta}$ such that $\mu_{x}(\widetilde{U})=0$. If $U:=\widetilde{U} \cap \partial X_{\theta}$, then by compactness and minimality of $L_{\Gamma} \cap \partial X_{\theta}$ (see Theorem A in [Lin13] ) there exists a finite set $\Lambda \subset \Gamma$ such that

$$
L_{\Gamma} \cap \partial X_{\theta} \subset \bigcup_{\gamma \in \Lambda} \gamma U
$$

Moreover, by $\Gamma$-equivariance

$$
\mu_{x}\left(L_{\Gamma} \cap \partial X_{\theta}\right) \leq \sum_{\gamma \in \Lambda} \mu_{x}(\gamma U)=\sum_{\gamma \in \Lambda} \mu_{\gamma^{-1} x}(U) \leq \sum_{\gamma \in \Lambda} \mu_{\gamma^{-1} x}(\widetilde{U})=0
$$

since $\mu_{\gamma^{-1} x}, \gamma \in \Lambda$, is absolutely continuous with respect to $\mu_{x}$.

Recall the definition of the distance vector (2) from Section 3 .

Theorem 7.2 (Shadow lemma) Let $\mu$ be a $(b, \theta)$-density. Then there exists a constant $c_{0}>0$ such that for any $c>c_{0}$ there exists a constant $D(c)>1$ with the property

$$
\frac{1}{D(c)} \mathrm{e}^{-\langle b, H(o, \gamma o)\rangle} \leq \mu_{o}\left(S h\left(o: B_{\gamma o}(c)\right)\right) \leq D(c) \mathrm{e}^{-\langle b, H(o, \gamma o)\rangle}
$$

for all $\gamma \in \Gamma$ with $d(o, \gamma o)>c$.

Proof. For $i \in\{1,2, \ldots, r\}$ we let $U_{i} \subset \partial X_{i}$ be open neighborhoods of $h_{i}^{+}, \Lambda \subset \Gamma$ a finite set and $c_{0}>0$ such that the assertion of Proposition 3.2 holds. If $U_{\theta}$ denotes the Cartesian product of the sets $U_{i}$ with $i \in I^{+}(\theta)$, then for all $\lambda \in \Lambda$ the set

$$
\lambda\left(U_{\theta} \times\{\theta\}\right) \subset \partial X_{\theta}
$$

is a relatively open neighborhood of a limit point in $\partial X_{\theta}$, so by the previous lemma

$$
q:=\min \left\{\mu_{o}\left(\lambda\left(U_{\theta} \times\{\theta\}\right)\right): \lambda \in \Lambda\right\}
$$


is strictly positive. Moreover, if $c \geq c_{0}$ and $\gamma \in \Gamma$ such that $d(o, \gamma o)>c$ then by Proposition 3.2 there exists $\lambda \in \Lambda$ such that $\lambda\left(U_{\theta} \times\{\theta\}\right) \subset \operatorname{Sh}\left(\gamma^{-1} o: B_{o}(c)\right)$. Hence for $c \geq c_{0}$ and $\gamma \in \Gamma$ with $d(o, \gamma o)>c$ we have

$$
\mu_{o}(\partial X) \geq \mu_{o}\left(\operatorname{Sh}\left(\gamma^{-1} o: B_{o}(c)\right)\right) \geq q>0 .
$$

Put $S_{\gamma}:=\operatorname{Sh}\left(o: B_{\gamma o}(c)\right)$ and recall the definition of the Busemann vector (11). The properties (ii) and (iii) of a $(b, \theta)$-density imply

$$
\begin{aligned}
\mu_{o}\left(\operatorname{Sh}\left(\gamma^{-1} o: B_{o}(c)\right)\right) & =\mu_{o}\left(\gamma^{-1} S_{\gamma}\right)=\mu_{\gamma o}\left(S_{\gamma}\right) \\
& =\int_{S_{\gamma}} d \mu_{\gamma o}(\tilde{\eta})=\int_{\operatorname{Sh}\left(o: B_{\gamma o}(c)\right)} \mathrm{e}^{\left\langle b, \mathcal{B}_{\tilde{\eta}}(o, \gamma o)\right\rangle} d \mu_{o}(\tilde{\eta})
\end{aligned}
$$

By Lemma 3.8,

$$
\mathrm{e}^{-2 c} \mathrm{e}^{\langle b, H(o, \gamma o)\rangle} \mu_{o}\left(S_{\gamma}\right)<\mu_{o}\left(\operatorname{Sh}\left(\gamma^{-1} o: B_{o}(c)\right)\right) \leq \mathrm{e}^{\langle b, H(o, \gamma o)\rangle} \mu_{o}\left(S_{\gamma}\right),
$$

so equation (29) allows us to conclude

$$
\mathrm{e}^{-\langle b, H(o, \gamma o)\rangle} q \leq \mu_{o}\left(S_{\gamma}\right) \leq \mathrm{e}^{-\langle b, H(o, \gamma o)\rangle} \mathrm{e}^{2 c} \cdot \mu_{o}(\partial X) .
$$

The following applications of Theorem 7.2 yield relations between the exponent of growth of a given slope $\theta \in E$ and the parameters of a $(b, \theta)$-density.

Theorem 7.3 If for $\theta \in E^{+} a \Gamma$-invariant $(b, \theta)$-density exists, then

$$
\delta_{\theta}(\Gamma) \leq\langle b, \theta\rangle .
$$

Proof. Suppose $\mu$ is a $(b, \theta)$-density. Let $c>c_{0}+1$, where $c_{0}>0$ is as in Theorem 7.2 , $\varepsilon>0$ and $n \in \mathbb{N}, n>3 c_{0}$ arbitrary. Let $\tilde{\eta}=\left(\eta_{1}, \eta_{2}, \ldots, \eta_{r}, \theta\right) \in \operatorname{supp}\left(\mu_{o}\right)$. We only need $N(\varepsilon) n^{r-1}$ balls of radius 1 in $X$ to cover the set

$$
\left\{\left(\sigma_{o_{1}, \eta_{1}}\left(t \hat{\theta}_{1}\right), \sigma_{o_{2}, \eta_{2}}\left(t \hat{\theta}_{2}\right), \ldots, \sigma_{o_{r}, \eta_{r}}\left(t \hat{\theta}_{r}\right)\right) \in X: n-1 \leq t<n,\|\hat{\theta}-\theta\|<\varepsilon\right\},
$$

and $N(\varepsilon)$ is independent of $n$. Since $\Gamma$ is discrete, a $2 c$-neighborhood of any of these balls contains a uniformly bounded number $M_{c}$ of elements of $\Gamma \cdot o$. Hence every point in the support of $\mu_{o}$ is contained in at most $M_{c} N(\varepsilon) n^{r-1}$ Weyl chamber shadows $\operatorname{Sh}\left(o: B_{\gamma o}(c)\right)$ with $\gamma \in \Gamma^{\prime}:=\{\gamma \in \Gamma:\|\widehat{H}(o, \gamma o)-\theta\|<\varepsilon, n-1 \leq d(o, \gamma o)<n\}$. Therefore

$$
\begin{aligned}
\sum_{\gamma \in \Gamma^{\prime}} \mu_{o}\left(\operatorname{Sh}\left(o: B_{\gamma o}(c)\right)\right) & \leq M_{c} N(\varepsilon) n^{r-1} \cdot \mu_{o}\left(\bigcup_{\gamma \in \Gamma^{\prime}} \operatorname{Sh}\left(o: B_{\gamma o}(c)\right)\right) \\
& \leq M_{c} N(\varepsilon) n^{r-1} \cdot \mu_{o}\left(\partial X_{\theta}\right)=M_{c} N(\varepsilon) n^{r-1} \cdot \mu_{o}(\partial X) .
\end{aligned}
$$

Furthermore, if $\gamma \in \Gamma^{\prime}$ then $\widehat{H}(o, \gamma o) \in E$ satisfies $\|\widehat{H}(o, \gamma o)-\theta\| \leq \varepsilon$. Using the Cauchy-Schwarz inequality we get for $\gamma \in \Gamma^{\prime}$

$$
\langle b, \widehat{H}(o, \gamma o)\rangle=\langle b, \theta\rangle+\langle b, \widehat{H}(o, \gamma o)-\theta\rangle \leq\langle b, \theta\rangle+\|b\| \varepsilon .
$$


With the notation

$$
\Delta N_{\theta}^{\varepsilon}(o, o ; n):=\#\{\gamma \in \Gamma: n-1<d(o, \gamma o) \leq n,\|\widehat{H}(o, \gamma o)-\theta\|<\varepsilon\}
$$

from Section 4 and the shadow lemma Theorem 7.2 we conclude

$$
\begin{aligned}
\Delta N_{\theta}^{\varepsilon}(o, o ; n) \frac{1}{D(c)} \mathrm{e}^{-\langle b, \theta\rangle n} \leq \sum_{\gamma \in \Gamma^{\prime}} \frac{1}{D(c)} \mathrm{e}^{-\langle b, H(o, \gamma o)\rangle+\varepsilon\|b\| d(o, \gamma o)} \\
\quad \leq \mathrm{e}^{\varepsilon\|b\| n} \sum_{\gamma \in \Gamma^{\prime}} \mu_{o}\left(\operatorname{Sh}\left(o: B_{\gamma o}(c)\right)\right) \leq \mathrm{e}^{\varepsilon\|b\| n} M_{c} N(\varepsilon) n^{r-1} \cdot \mu_{o}(\partial X) .
\end{aligned}
$$

Using (15) we therefore get

$$
\delta_{\theta}^{\varepsilon}(o, o) \leq \limsup _{n \rightarrow \infty} \frac{1}{n} \ln \left(D(c) M_{c} N(\varepsilon) n^{r-1} \cdot \mu_{o}(\partial X) \mathrm{e}^{\langle b, \theta\rangle n+\varepsilon\|b\| n}\right)=\langle b, \theta\rangle+\varepsilon\|b\|
$$

and the claim follows as $\varepsilon \searrow 0$.

We remark that the proof of the above proposition does not work for $\theta \in E \backslash E^{+}$ because a singular boundary point can be contained in infinitely many Weyl chamber shadows $\operatorname{Sh}\left(o: B_{\gamma o}(c)\right)$ with $\gamma \in\{\gamma \in \Gamma:\|\widehat{H}(o, \gamma o)-\theta\|<\varepsilon, n-1 \leq d(o, \gamma o)<n\}$. This is due to the fact that the Weyl chambers $\mathcal{C}_{o, \tilde{\eta}}$ with $\tilde{\eta} \in \partial X^{\operatorname{sing}}$ are too big.

We next recall the notion of radial limit point from Definition 1.3 of the introduction. If $\theta \in E$ then using the equality (8) we can describe the radial limit set in $\partial X_{\theta}$ via

$$
L_{\Gamma}^{r a d} \cap \partial X_{\theta}=\bigcup_{c>0} \bigcap_{R>c} \bigcap_{\varepsilon>0} \bigcup_{\substack{\gamma \in \Gamma \\ d(o, \gamma o)>R \\\|\widehat{H}(o, \gamma o)-\theta\|<\varepsilon}} \operatorname{Sh}\left(o: B_{\gamma o}(c)\right) \cap \partial X_{\theta}
$$

Together with the previous theorem the following statement says that if a $(b, \theta)$-density gives positive measure to the regular radial limit set, then the exponent of growth of $\Gamma$ of slope $\theta$ is completely determined by the parameters $b=\left(b_{1}, b_{2}, \ldots, b_{r}\right)$.

Theorem 7.4 If $\theta \in E$ and $a(b, \theta)$-density gives positive measure to $L_{\Gamma}^{\text {rad }}$, then $\delta_{\theta}(\Gamma) \geq\langle b, \theta\rangle$.

Proof. Suppose $\mu$ is a $(b, \theta)$-density such that $\mu_{o}\left(L_{\Gamma}^{r a d}\right)>0$. Since $\operatorname{supp}\left(\mu_{o}\right) \subset \partial X_{\theta}$ this implies $\mu_{o}\left(L_{\Gamma}^{r a d} \cap \partial X_{\theta}\right)>0$. By definition (30) there exists $c>0$ such that with

$$
L_{\Gamma}^{r a d}(c) \cap \partial X_{\theta}:=\bigcap_{R>c} \bigcap_{\varepsilon>0} \bigcup_{\substack{\gamma \in \Gamma \\ d(o, \gamma o)>R \\\|\hat{H}(o, \gamma o)-\theta\|<\varepsilon}} \operatorname{Sh}\left(o: B_{\gamma o}(c)\right) \cap \partial X_{\theta}
$$

we have $\mu_{o}\left(L_{\Gamma}^{\mathrm{rad}}(c) \cap \partial X_{\theta}\right)>0$. Without loss of generality we may assume that $c>c_{0}$ with $c_{0}>0$ as in Theorem 7.2 . Let $\varepsilon>0$ and $R>c$ arbitrary, and set

$$
\Gamma^{\prime}:=\{\gamma \in \Gamma: d(o, \gamma o)>R,\|\widehat{H}(o, \gamma o)-\theta\|<\varepsilon\} .
$$


Then by (31)

$$
L_{\Gamma}^{r a d}(c) \cap \partial X_{\theta} \subset \bigcup_{\gamma \in \Gamma^{\prime}} \operatorname{Sh}\left(o: B_{\gamma o}(c)\right) \cap \partial X_{\theta},
$$

and we estimate

$$
\begin{aligned}
0<\mu_{o}\left(L_{\Gamma}^{r a d}(c)\right) & =\mu_{o}\left(L_{\Gamma}^{r a d}(c) \cap \partial X_{\theta}\right) \\
& \leq \sum_{\gamma \in \Gamma^{\prime}} \mu_{o}\left(\operatorname{Sh}\left(o: B_{\gamma o}(c)\right)\right) \leq D(c) \sum_{\gamma \in \Gamma^{\prime}} \mathrm{e}^{-\langle b, H(o, \gamma o)\rangle} .
\end{aligned}
$$

This implies that for any $\varepsilon>0$ the tail of the series

$$
\sum_{\substack{\gamma \in \Gamma \\\|\widehat{H}(o, \gamma o)-\theta\|<\varepsilon}} \mathrm{e}^{-\langle b, H(o, \gamma o)\rangle}
$$

does not tend to zero. Therefore the sum above diverges, and by Proposition 4.1 (b) there exists $\hat{\theta} \in E,\|\hat{\theta}-\theta\| \leq \varepsilon$ such that

$$
\langle b, \hat{\theta}\rangle \leq \delta_{\hat{\theta}}(\Gamma) .
$$

Taking the limit as $\varepsilon \searrow 0$, we conclude $\langle b, \theta\rangle \leq \delta_{\theta}(\Gamma)$.

Recall the definition of the Busemann vector (11) from Section 3. The following two lemmata hold for any $\theta \in E$ and will be important for the proof of Theorem 7.7 .

Lemma 7.5 Let $\mu$ be a $(b, \theta)$-density. If $\tilde{\eta} \in \partial X_{\theta}$ is a point mass for $\mu$, and $\Gamma_{\tilde{\eta}}$ its stabilizer, then for any $\gamma \in \Gamma_{\tilde{\eta}}$ and $x=\left(x_{1}, x_{2}, \ldots, x_{r}\right) \in X$ we have

$$
\left\langle b, \mathcal{B}_{\tilde{\eta}}(x, \gamma x)\right\rangle=\sum_{i=1}^{r} b_{i} \beta_{\eta_{i}}\left(x_{i}, \gamma_{i} x_{i}\right)=0 .
$$

In particular, if $\gamma, \hat{\gamma} \in \Gamma$ are representatives of the same coset in $\Gamma / \Gamma_{\tilde{\eta}}$, then

$$
\left\langle b, \mathcal{B}_{\tilde{\eta}}\left(x, \gamma^{-1} x\right)\right\rangle=\left\langle b, \mathcal{B}_{\tilde{\eta}}\left(x, \hat{\gamma}^{-1} x\right)\right\rangle .
$$

Proof. For $x \in X$ and $\gamma \in \Gamma_{\tilde{\eta}}$ arbitrary we have by $\Gamma$-equivariance

$$
\mu_{x}(\tilde{\eta})=\mu_{x}\left(\gamma^{-1} \tilde{\eta}\right)=\mu_{\gamma x}(\tilde{\eta})
$$

From the assumption that $\tilde{\eta}$ is a point mass and property (iii) in Definition 1.2 we get

$$
1=\frac{\mu_{\gamma x}(\tilde{\eta})}{\mu_{x}(\tilde{\eta})}=\mathrm{e}^{\left\langle b, \mathcal{B}_{\tilde{\eta}}(x, \gamma x)\right\rangle}
$$

so $\left\langle b, \mathcal{B}_{\tilde{\eta}}(x, \gamma x)\right\rangle=0$ for all $x \in X$ and all $\gamma \in \Gamma_{\tilde{\eta}}$.

Next let $\gamma, \hat{\gamma} \in \Gamma$ such that $\gamma \Gamma_{\tilde{\eta}}=\hat{\gamma} \Gamma_{\tilde{\eta}} \in \Gamma / \Gamma_{\tilde{\eta}}$. Then $\hat{\gamma}^{-1} \gamma \in \Gamma_{\tilde{\eta}}$ and therefore $\left\langle b, \mathcal{B}_{\tilde{\eta}}\left(\gamma^{-1} x,\left(\hat{\gamma}^{-1} \gamma\right) \gamma^{-1} x\right)=0\right.$; using the cocycle identity for the Busemann vector we get

$$
\begin{aligned}
\left\langle b, \mathcal{B}_{\tilde{\eta}}\left(x, \gamma^{-1} x\right)\right\rangle & =\left\langle b, \mathcal{B}_{\tilde{\eta}}\left(x, \gamma^{-1} x\right)\right\rangle+\left\langle b, \mathcal{B}_{\tilde{\eta}}\left(\gamma^{-1} x, \hat{\gamma}^{-1} \gamma \gamma^{-1} x\right)\right\rangle \\
& =\left\langle b, \mathcal{B}_{\tilde{\eta}}\left(x, \hat{\gamma}^{-1} \gamma \gamma^{-1} x\right)\right\rangle=\left\langle b, \mathcal{B}_{\tilde{\eta}}\left(x, \hat{\gamma}^{-1} x\right)\right\rangle
\end{aligned}
$$


Lemma 7.6 If $\tilde{\eta} \in \partial X_{\theta}$ is a point mass for a $(b, \theta)$-density $\mu$, then the sum

$$
\sum \mathrm{e}^{\left\langle b, \mathcal{B}_{\tilde{\eta}}\left(o, \gamma^{-1} o\right)\right\rangle}
$$

taken over a system of coset representatives of $\Gamma / \Gamma_{\tilde{\eta}}$ converges.

Proof. If $\gamma$ and $\hat{\gamma}$ are representatives of different cosets in $\Gamma / \Gamma_{\tilde{\eta}}$, then $\gamma \tilde{\eta} \neq \hat{\gamma} \tilde{\eta}$ and hence, by $\Gamma$-equivariance, the sum $\sum \mu_{\gamma^{-1} o}(\tilde{\eta})=\sum \mu_{o}(\gamma \tilde{\eta})$ over a system of coset representatives of $\Gamma / \Gamma_{\tilde{\eta}}$ is bounded above by $\mu_{o}(\partial X)$. By property (iii) in Definition 1.2 and the assumption that $\tilde{\eta}$ is a point mass we conclude that the sum

$$
\sum \mathrm{e}^{\left\langle b, \mathcal{B}_{\tilde{\eta}}\left(o, \gamma^{-1} o\right)\right\rangle}=\sum \frac{\mu_{\gamma^{-1} o}(\tilde{\eta})}{\mu_{o}(\tilde{\eta})}=\frac{1}{\mu_{o}(\tilde{\eta})} \sum \mu_{\gamma^{-1} o}(\tilde{\eta})
$$

over a system of coset representatives of $\Gamma / \Gamma_{\eta}$ is bounded above by $\frac{\mu_{o}(\partial X)}{\mu_{o}(\tilde{\eta})}$. Since $\mu_{o}$ is a finite measure and $\mu_{o}(\tilde{\eta})>0$, the above sum converges.

Theorem 7.7 If $\delta_{\theta}(\Gamma)>0$ then a regular radial limit point $\tilde{\eta} \in L_{\Gamma}^{\text {rad }} \cap \partial X^{\text {reg }}$ is not a point mass for any $(b, \theta)$-density.

Proof. Let $\mu$ be a $(b, \theta)$-density. If $\tilde{\eta} \notin \partial X_{\theta}$, then $\tilde{\eta} \notin \operatorname{supp}\left(\mu_{o}\right)$, hence $\tilde{\eta}$ cannot be a point mass. In particular, it suffices to consider $\theta \in E^{+}$.

Suppose $\tilde{\eta}=\left(\eta_{1}, \eta_{2}, \ldots, \eta_{r}, \theta\right) \in L_{\Gamma}^{r a d} \cap \partial X_{\theta} \subset \partial X^{r e g}$ is a point mass for $\mu$. Then by Theorem 7.4 we have $\langle b, \theta\rangle=\delta_{\theta}(\Gamma)>0$, hence by continuity of the map $E \rightarrow \mathbb{R}, \hat{\theta} \mapsto\langle b, \hat{\theta}\rangle$ there exists $\varepsilon>0$ such that every $\hat{\theta} \in E$ with $\|\hat{\theta}-\theta\|<\varepsilon$ satisfies

$$
\langle b, \hat{\theta}\rangle \geq \frac{\delta_{\theta}(\Gamma)}{2}>0 .
$$

Moreover, by the formula (30) for the radial limit set in $\partial X_{\theta}$ there exists a constant $c>0$ and a sequence $\left(\gamma_{n}\right)=\left(\left(\gamma_{n, 1}, \gamma_{n, 2}, \ldots, \gamma_{n, r}\right)\right) \subset \Gamma$ such that $\left\|\widehat{H}\left(o, \gamma_{n} o\right)-\theta\right\|<\varepsilon$ and $\tilde{\eta} \in S\left(o: B_{\gamma_{n} o}(c)\right)$ for all $n \in \mathbb{N}$. Corollary 3.8 implies

$$
\beta_{\eta_{i}}\left(o_{i}, \gamma_{n, i} o_{i}\right)>d_{i}\left(o_{i}, \gamma_{n, i} o_{i}\right)-2 c \quad \text { for all } n \in \mathbb{N} \text { and all } i \in\{1,2, \ldots, r\},
$$

and by choice of $\varepsilon>0$ we have $\left\langle b, \widehat{H}\left(o, \gamma_{n} o\right)\right\rangle \geq q$ for all $n \in \mathbb{N}$. Summarizing we conclude

$$
\left\langle b, \mathcal{B}_{\tilde{\eta}}\left(o, \gamma_{n} o\right)\right\rangle>\left\langle b, H\left(o, \gamma_{n} o\right)\right\rangle-2\|b\| c \geq q \cdot d\left(o, \gamma_{n} o\right)-2\|b\| c \quad \longrightarrow \infty \quad \text { as } \quad n \rightarrow \infty .
$$

Passing to a subsequence if necessary we may therefore assume that $\left\langle b, \mathcal{B}_{\tilde{\eta}}\left(o, \gamma_{n} o\right)\right\rangle$ is strictly increasing to infinity as $n \rightarrow \infty$.

Now suppose there exist $l, j \in \mathbb{N}, l \neq j$ such that $\gamma_{l}^{-1} \Gamma_{\tilde{\eta}}=\gamma_{j}^{-1} \Gamma_{\tilde{\eta}}$. Since $\tilde{\eta}$ is a point mass for $\mu$, Lemma 7.5 implies

$$
\left\langle b, \mathcal{B}_{\tilde{\eta}}\left(o, \gamma_{j} o\right)\right\rangle=\left\langle b, \mathcal{B}_{\tilde{\eta}}\left(o, \gamma_{l} o\right)\right\rangle,
$$


in contradiction to the choice of the subsequence $\left(\gamma_{n}\right)$. Hence $\gamma_{l}^{-1} \Gamma_{\tilde{\eta}} \neq \gamma_{j}^{-1} \Gamma_{\tilde{\eta}}$ for all $l \neq j$, and the sum $\sum \mathrm{e}^{\left\langle b, \mathcal{B}_{\tilde{\eta}}(o, \gamma o)\right\rangle}$ over a system of coset representatives of $\Gamma / \Gamma_{\tilde{\eta}}$ is bounded below by

$$
\sum_{n \in \mathbb{N}} \mathrm{e}^{\left\langle b, \mathcal{B}_{\tilde{\eta}}\left(o, \gamma_{n} o\right)\right\rangle}
$$

The divergence of this series yields a contradiction to Lemma 7.6 and we conclude that $\tilde{\eta}$ cannot be a point mass for $\mu_{o}$.

\section{References}

[Alb99] P. Albuquerque, Patterson-Sullivan theory in higher rank symmetric spaces, Geom. Funct. Anal. 9 (1999), no. 1, 1-28.

[Bal82] Werner Ballmann, Axial isometries of manifolds of nonpositive curvature, Math. Ann. 259 (1982), no. 1, 131-144.

[Bal95] Werner Ballmann, Lectures on spaces of nonpositive curvature, DMV Seminar, vol. 25, Birkhäuser Verlag, Basel, 1995, With an appendix by Misha Brin.

[BB95] Werner Ballmann and Michael Brin, Orbihedra of nonpositive curvature, Inst. Hautes Études Sci. Publ. Math. (1995), no. 82, 169-209 (1996).

[BGS85] Werner Ballmann, Mikhael Gromov, and Viktor Schroeder, Manifolds of nonpositive curvature, Progress in Mathematics, vol. 61, Birkhäuser Boston Inc., Boston, MA, 1985.

[BH99] Martin R. Bridson and André Haefliger, Metric spaces of non-positive curvature, Grundlehren der Mathematischen Wissenschaften [Fundamental Principles of Mathematical Sciences], vol. 319, Springer-Verlag, Berlin, 1999.

[Bur93] Marc Burger, Intersection, the Manhattan curve, and Patterson-Sullivan theory in rank 2, Internat. Math. Res. Notices (1993), no. 7, 217-225.

[CF10] Pierre-Emmanuel Caprace and Koji Fujiwara, Rank-one isometries of buildings and quasi-morphisms of Kac-Moody groups, Geom. Funct. Anal. 19 (2010), no. 5, 1296-1319.

[CR09] Pierre-Emmanuel Caprace and Bertrand Rémy, Simplicity and superrigidity of twin building lattices, Invent. Math. 176 (2009), no. 1, 169-221.

[CS11] Pierre-Emmanuel Caprace and Michah Sageev, Rank rigidity for CAT(0) cube complexes, Geom. Funct. Anal. 21 (2011), no. 4, 851-891.

[DK08] Françoise Dal'Bo and Inkang Kim, Shadow lemma on the product of Hadamard manifolds and applications, Actes du Séminaire de Théorie Spectrale et Géométrie. Vol. 25. Année 2006-2007, Sémin. Théor. Spectr. Géom., vol. 25, Univ. Grenoble I, Saint, 2008, pp. 105-119. 
[Kni97] G. Knieper, On the asymptotic geometry of nonpositively curved manifolds, Geom. Funct. Anal. 7 (1997), no. 4, 755-782.

[Lin04] Gabriele Link, Hausdorff dimension of limit sets of discrete subgroups of higher rank Lie groups, Geom. Funct. Anal. 14 (2004), no. 2, 400-432.

[Lin07] Gabriele Link, Asymptotic geometry and growth of conjugacy classes of nonpositively curved manifolds, Ann. Global Anal. Geom. 31 (2007), no. 1, 37-57.

[Lin10] Gabriele Link, Asymptotic geometry in products of Hadamard spaces with rank one isometries, Geom. Topol. 14 (2010), no. 2, 1063-1094.

[Lin13] G. Link, Asymptotic geometry in higher products of rank one Hadamard spaces, http://arxiv.org/abs/1308.5584, 2013.

[Pat76] S. J. Patterson, The limit set of a Fuchsian group, Acta Math. 136 (1976), no. $3-4,241-273$.

[Qui02] J.-F. Quint, Mesures de Patterson-Sullivan en rang supérieur, Geom. Funct. Anal. 12 (2002), no. 4, 776-809.

[Rém99] Bertrand Rémy, Construction de réseaux en théorie de Kac-Moody, C. R. Acad. Sci. Paris Sér. I Math. 329 (1999), no. 6, 475-478.

[Sul79] Dennis Sullivan, The density at infinity of a discrete group of hyperbolic motions, Inst. Hautes Études Sci. Publ. Math. (1979), no. 50, 171-202.

Gabriele Link

Institut für Algebra und Geometrie

Karlsruher Institut für Technologie (KIT)

Kaiserstr. 89-93

D-76133 Karlsruhe

e-mail: gabriele.link@kit.edu 